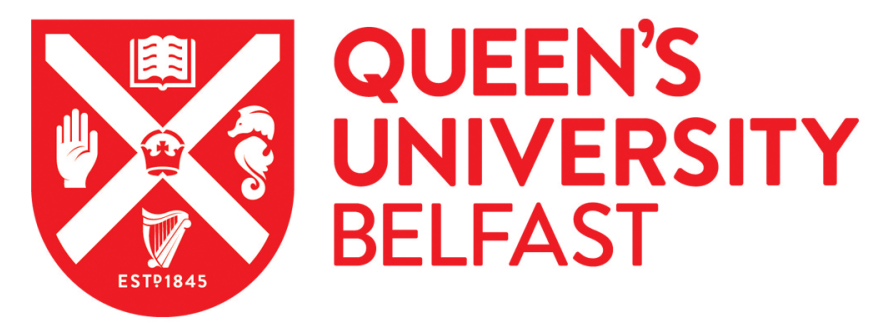

\title{
Numerical modeling of laser-driven experiments aiming to demonstrate magnetic field amplification via turbulent dynamo
}

Tzeferacos, P., Rigby, A., Bott, A., Casner, A., Cattaneo, F., Churazov, E. M., Emig, J., Flocke, N., Fiuza, F., Forest, C. B., Graziani, C., Katz, J., Koenig, M., Meinecke, J., Petrasso, R., Park, H-S., Remington, B. A., Ross, J. S., Ryu, D., ... Lamb, D. Q. (2017). Numerical modeling of laser-driven experiments aiming to demonstrate magnetic field amplification via turbulent dynamo. Physics of Plasmas, 24(4), 041404.

https://doi.org/10.1063/1.4978628

Published in:

Physics of Plasmas

Document Version:

Publisher's PDF, also known as Version of record

Queen's University Belfast - Research Portal:

Link to publication record in Queen's University Belfast Research Portal

Publisher rights

Copyright 2018 AIP. This work is made available online in accordance with the publisher's policies. Please refer to any applicable terms of use of the publisher

General rights

Copyright for the publications made accessible via the Queen's University Belfast Research Portal is retained by the author(s) and / or other copyright owners and it is a condition of accessing these publications that users recognise and abide by the legal requirements associated with these rights.

Take down policy

The Research Portal is Queen's institutional repository that provides access to Queen's research output. Every effort has been made to ensure that content in the Research Portal does not infringe any person's rights, or applicable UK laws. If you discover content in the Research Portal that you believe breaches copyright or violates any law, please contact openaccess@qub.ac.uk. 


\section{Numerical modeling of laser-driven experiments aiming to demonstrate magnetic field amplification via turbulent dynamo}

P. Tzeferacos, A. Rigby, A. Bott, A. R. Bell, R. Bingham, A. Casner, F. Cattaneo, E. M. Churazov, J. Emig, N. Flocke, F. Fiuza, C. B. Forest, J. Foster, C. Graziani, J. Katz, M. Koenig, C.-K. Li, J. Meinecke, R. Petrasso, H.-S. Park, B. A. Remington, J. S. Ross, D. Ryu, D. Ryutov, K. Weide, T. G. White, B. Reville, F. Miniati, A. A. Schekochihin, D. H. Froula, G. Gregori, and D. Q. Lamb

Citation: Physics of Plasmas 24, 041404 (2017); doi: 10.1063/1.4978628

View online: https://doi.org/10.1063/1.4978628

View Table of Contents: http://aip.scitation.org/toc/php/24/4

Published by the American Institute of Physics

\section{Articles you may be interested in}

Magnetic field production via the Weibel instability in interpenetrating plasma flows

Physics of Plasmas 24, 041410 (2017); 10.1063/1.4982044

Particle acceleration in laser-driven magnetic reconnection

Physics of Plasmas 24, 041408 (2017); 10.1063/1.4978627

Formation of high-speed electron jets as the evidence for magnetic reconnection in laser-produced plasma Physics of Plasmas 24, 041406 (2017); 10.1063/1.4978883

On the generation of magnetized collisionless shocks in the large plasma device Physics of Plasmas 24, 041405 (2017); 10.1063/1.4978882

A self-consistent analytical model for the upstream magnetic-field and ion-beam properties in Weibel-mediated collisionless shocks

Physics of Plasmas 24, 041409 (2017); 10.1063/1.4979187

Development of an inertial confinement fusion platform to study charged-particle-producing nuclear reactions relevant to nuclear astrophysics

Physics of Plasmas 24, 041407 (2017); 10.1063/1.4979186

\section{PHYSICS TODAY}

MANAGER'S GUIDE

WHITEPAPERS
Accelerate R\&D with Multiphysics Simulation
READ NOW

PRESENTED BY

๑๐ $\subset$ MSOL 


\title{
Numerical modeling of laser-driven experiments aiming to demonstrate magnetic field amplification via turbulent dynamo
}

\author{
P. Tzeferacos, ${ }^{1,2, a)}$ A. Rigby, ${ }^{2}$ A. Bott, ${ }^{2}$ A. R. Bell, ${ }^{2}$ R. Bingham,,${ }^{3,4}$ A. Casner, ${ }^{5}$ F. Cattaneo, ${ }^{1}$ \\ E. M. Churazov, ${ }^{6,7}$ J. Emig, ${ }^{8}$ N. Flocke, ${ }^{1}$ F. Fiuza, ${ }^{9}$ C. B. Forest,${ }^{10}$ J. Foster,${ }^{11}$ C. Graziani, ${ }^{1}$ \\ J. Katz, ${ }^{12}$ M. Koenig, ${ }^{13}$ C.-K. Li, ${ }^{14}$ J. Meinecke,${ }^{2}$ R. Petrasso, ${ }^{14}$ H.-S. Park, ${ }^{8}$

(Received 24 October 2016; accepted 3 February 2017; published online 22 March 2017)

The universe is permeated by magnetic fields, with strengths ranging from a femtogauss in the voids between the filaments of galaxy clusters to several teragauss in black holes and neutron stars. The standard model behind cosmological magnetic fields is the nonlinear amplification of seed fields via turbulent dynamo to the values observed. We have conceived experiments that aim to demonstrate and study the turbulent dynamo mechanism in the laboratory. Here, we describe the design of these experiments through simulation campaigns using FLASH, a highly capable radiation magnetohydrodynamics code that we have developed, and large-scale three-dimensional simulations on the Mira supercomputer at the Argonne National Laboratory. The simulation results indicate that the experimental platform may be capable of reaching a turbulent plasma state and determining the dynamo amplification. We validate and compare our numerical results with a small subset of experimental data using synthetic diagnostics. Published by AIP Publishing.

[http://dx.doi.org/10.1063/1.4978628]

\section{INTRODUCTION}

Magnetic fields are encountered throughout the universe. $^{1}$ Observational methods based on Faraday rotation and polarization measurements, Zeeman effect, and magneto-bremsstrahlung, even in situ measurements in the case of proximal astrophysical objects, have revealed the broad range of values of cosmical magnetic fields: ${ }^{2}$ from a femtogauss in the tenuous voids between galaxy cluster filaments, to several microgauss in galaxies and galaxy clusters, a milligauss in molecular clouds, a few gauss in planets, tens of kilogauss in ordinary stars and accretion disks, a megagauss in white dwarfs, and many teragauss in the vicinity of black holes and neutron stars. Astrophysical fields are often "strong," in the sense that their energy can amount to a substantial fraction of system's energy budget, making them

\footnotetext{
${ }^{\text {a)}}$ Electronic mail: petros.tzeferacos@flash.uchicago.edu
}

salient agents in astrophysical and cosmological phenomena. This, in conjunction with their ubiquity, has led naturally to the two-fold question of their origin: (1) how are magnetic fields generated and (2) how do they reach and maintain such large values?

The answer to this question is commonly expressed in terms of dynamo action that operates on seed magnetic fields. ${ }^{1,3,4}$ Cosmological seed magnetic fields can be generated via a number of mechanisms, such as plasma instabilities and thermal electromotive forces, ${ }^{2}$ the Biermann battery effect ${ }^{5}$ that arises from misaligned electron pressure and density gradients, or the Weibel instability ${ }^{6}$ that can occur in collisionless shocks. ${ }^{7}$ These seed fields are then amplified by the hydromagnetic dynamo mechanism which achieves a sustained conversion of kinetic energy into magnetic energy throughout the bulk of an electrically conducting fluid. This mechanism was first invoked almost a century ago for solar magnetic fields. ${ }^{8}$ 
An attractive feature of dynamos is that the requirements for their operation are modest. The two key ingredients are fluid motions that are not too symmetric ${ }^{9,10}$ and high electrical conductivity. ${ }^{11,12}$ Both of these requirements are amply satisfied by the turbulent motions and high magnetic Reynolds numbers prevalent in most astrophysical situations, ${ }^{2}$ supporting the expectation that dynamo action is widespread in astrophysics. ${ }^{1,2,13}$ While astrophysical dynamos come in many flavors, ${ }^{14}$ they are often distinguished ${ }^{15}$ between large-scale (or mean-field) dynamos, in which the magnetic field grows at scales larger than those of the fluid motion, and small-scale (or fluctuation) dynamos, where the growth occurs at or below the outer scales of motion. In this article, we will concern ourselves with small-scale dynamo, at magnetic Prandtl numbers (i.e., magnetic-to-fluid Reynolds number ratio) smaller than unity. ${ }^{15}$ Astrophysical environments with small magnetic Prandtl numbers include planetary cores, stellar convection zones, the galactic disk, and parts of the interstellar medium. ${ }^{2}$

Even though conditions favorable for dynamos are common in astrophysics, they are extremely difficult to realize in laboratory experiments. ${ }^{16}$ Thus, so far, our physical intuition in the working of dynamos is mostly based on theoretical considerations and numerical modeling. ${ }^{14,17-21}$ The reasons for this state of affairs can be easily explained. The two natural working fluids for laboratory dynamo experiments are liquid metals $^{22-24}$ and strongly ionized gases, i.e., plasmas. The electrical conductivity of liquid metals, however, makes reaching high magnetic Reynolds numbers difficult. Conversely, hot plasmas are much better electrical conductors, thus capable of reaching high magnetic Reynolds numbers, but they tend to be magnetically confined in fusion devices ${ }^{25}$ with gas-to-magnetic pressure ratios $\beta \ll 1$, thus unsuitable to study how they became strongly magnetized in the first place. Ideally, the aim should be to produce an initially low-magnetization plasma at high magnetic Reynolds numbers that can, in principle, be used to study the dynamo action in the laboratory. This approach, if successful, could provide a much-needed experimental component to the study of dynamos.

The advent of high-power lasers has opened a new field of research where, using simple scaling relations, ${ }^{26,27}$ astrophysical environments can be reproduced in the laboratory. ${ }^{28,29}$ The similarity achieved is sufficiently close to make such experiments relevant and informative, in terms of enabling the demonstration and study of the fundamental physical processes in play.

We have conceived experiments that aim to achieve turbulent dynamo in the laboratory. The results of these experiments are discussed in Ref. 30. In this paper, we describe the design of the experiments through simulation campaigns using FLASH, a radiation-magnetohydrodynamics (MHD) code that we have developed, large-scale three-dimensional simulations on the Mira supercomputer at the Argonne National Laboratory (ANL), and the validation of these simulations using a subset of the experimental data. Threedimensional simulations were required in order to represent with high fidelity both the geometry of the targets and key physical processes, so as to be predictive. The simulations were vital to ensure that the experiments achieved the strong turbulence and large magnetic Reynolds numbers needed for turbulent dynamo to operate. The simulations were also necessary to determine when to fire the diagnostics since the experiments last tens of nanoseconds, but the strongly amplified magnetic fields persisted for only a fraction of this time.

In Sec. II, we describe the high energy density laboratory plasma (HEDLP) capabilities of the FLASH code that was used in the simulations we performed. In Sec. III, we discuss the key elements of the platforms we used in previous experiments. These platforms informed the design of the experiments that we describe here. In Sec. IV, we describe the simulations that we performed and that led to the fielded experimental platform, as well as the final design. In Sec. V, we discuss the simulation results, as well as their validation against a subset of experimental data.

\section{SIMULATION CODE}

We use the FLASH code ${ }^{31,32}$ to carry out the large-scale simulations of our laser experiments to study the origin of cosmic magnetic fields. FLASH is a publicly available, ${ }^{33}$ parallel, multi-physics, adaptive mesh refinement (AMR), finite-volume Eulerian hydrodynamics, and MHD code. FLASH scales well to over 100,000 processors and uses a variety of parallelization techniques including domain decomposition, mesh replication, and threading to make optimal use of hardware resources.

Extensive HEDLP capabilities $^{34}$ have been added to FLASH, making it a suitable code for simulating laserdriven plasma experiments. The system of partial differential equations employed in the numerical modeling of the experiment has the general form

$$
\frac{\partial \mathbf{U}}{\partial t}+\nabla \cdot \mathbf{F}(\mathbf{U})=\mathbf{S}(\mathbf{U}),
$$

where $\mathbf{U}$ denotes the conserved variables (e.g., $\mathbf{U} \equiv(\rho, \mathbf{m}$, $\mathbf{B}, \mathcal{E})^{T}$ for ideal MHD), $\mathbf{F}(\mathbf{U})$ the fluxes, and $\mathbf{S}(\mathbf{U})$ the source terms. Here, we use the customary notation for density $(\rho)$, momentum density $(\mathbf{m})$, magnetic field $(\mathbf{B})$, and total energy density $(\mathcal{E})$.

A single-temperature ideal MHD formulation is insufficient to model HEDLP experiments: thermal equilibrium between electrons, ions, and radiation is disrupted by a number of physical processes, and equilibration times can be sufficiently long to warrant a multi-temperature treatment. To accomplish this, we extended the ideal MHD system of equations by retaining a single-fluid treatment while considering different temperatures for ions, electrons, and radiation (i.e., three temperatures or 3T). This extension requires that the total pressure be defined as $p_{t o t}=p_{i}+p_{e}+p_{r}+\mathbf{B}^{2} / 2$, where the subscripts $i, e$, and $r$ denote ions, electrons, and radiation, respectively. The continuity and momentum equations are given by

$$
\begin{gathered}
\frac{\partial \rho}{\partial t}+\nabla \cdot(\rho \mathbf{u})=0, \\
\frac{\partial \rho \mathbf{u}}{\partial t}+\nabla \cdot\left[\rho \mathbf{u} \mathbf{u}+p_{t o t} \mathbf{I}-\mathbf{B B}\right]=0 .
\end{gathered}
$$


In the induction equation, we consider the generalized form of Ohm's law $\mathbf{E}=-\mathbf{u} \times \mathbf{B}+\eta \mathbf{J}-\nabla p_{e} /\left(q_{e} n_{e}\right)$, where $q_{e}$ is the electron charge, $n_{e}$ the electron number density, $\eta$ the magnetic resistivity, ${ }^{35} \mathbf{J}=\nabla \times \mathbf{B}$ the current density, and $\mathbf{E}$ the electric field. Note that, in our isotropic treatment, we do not include the Nernst term ${ }^{36}$ because its effect is not important for the plasma conditions described here; it is however relevant in many other laser-based experiments. The induction equation then reads

$$
\frac{\partial \mathbf{B}}{\partial t}+\nabla \times[-\mathbf{u} \times \mathbf{B}]+\nabla \times\left[\eta \mathbf{J}-\frac{\nabla p_{e}}{q_{e} n_{e}}\right]=0,
$$

where the non-ideal terms on the left-hand side include magnetic diffusivity and the Biermann battery term. ${ }^{37,38} \mathrm{We}$ also evolve the total energy density equation

$$
\begin{aligned}
& \frac{\partial \mathcal{E}}{\partial t}+\nabla \cdot\left[\left(\mathcal{E}+p_{t o t}\right) \mathbf{u}-(\mathbf{u} \cdot \mathbf{B}) \mathbf{B}\right] \\
& -\nabla \cdot\left[\mathbf{B} \times\left(\eta \mathbf{J}-\frac{\nabla p_{e}}{q_{e} n_{e}}\right)\right]=-\nabla \cdot \mathbf{q}+S,
\end{aligned}
$$

where the total energy density is given by $\mathcal{E}=\rho E_{\text {tot }}=\rho e_{\text {int }}$ $+\rho \mathbf{u}^{2} / 2+\mathbf{B}^{2} / 2$ and the total specific internal energy includes the radiation energy, $e_{i n t}=e_{i}+e_{e}+e_{r}$. The total heat flux $\mathbf{q}=\mathbf{q}_{\mathbf{e}}+\mathbf{q}_{\mathbf{r}}$ is the sum of the electron heat flux $\mathbf{q}_{\mathbf{e}}=-\kappa \nabla T_{e}$ and the radiation flux $\mathbf{q}_{\mathbf{r}}$. For the former, we denote with $\kappa$ the electron conductivity ${ }^{39}$ and $T_{e}$ the electron temperature. The source term $S$ encompasses external contributions of energy, typically due to laser heating.

To treat the 3T components, we also consider the nonconservative energy equations for electrons, ions, and radiation. These can be written as

$$
\begin{gathered}
\frac{\partial \rho e_{i}}{\partial t}+\nabla \cdot\left(\rho e_{i} \mathbf{u}\right)+p_{i} \nabla \cdot \mathbf{u}=\rho \frac{c_{v, e}}{\tau_{e i}}\left(T_{e}-T_{i}\right) \\
\frac{\partial \rho e_{e}}{\partial t}+\nabla \cdot\left(\rho e_{e} \mathbf{u}\right)+p_{e} \nabla \cdot \mathbf{u}=\rho \frac{c_{v, e}}{\tau_{e i}}\left(T_{i}-T_{e}\right) \\
-\nabla \cdot \mathbf{q}_{e}+Q_{a b s}-Q_{e m i s}+Q_{l a s}+Q_{O h m}, \\
\frac{\partial \rho e_{r}}{\partial t}+\nabla \cdot\left(\rho e_{r} \mathbf{u}\right)+p_{r} \nabla \cdot \mathbf{u}=-\nabla \cdot \mathbf{q}_{r}-Q_{a b s}+Q_{e m i s},
\end{gathered}
$$

where $c_{v, e}$ is the electron specific heat, $\tau_{e i}$ the ion-electron relaxation time, $Q_{a b s}$ the rate of increase of the electron internal energy density due to radiation absorption, $Q_{e m i s}$ the rate of decrease due to radiation emission, and $Q_{O h m}$ the rate of increase due to Ohmic heating. The system closes with $3 \mathrm{~T}$ equations of state (EoS) that connect internal energies, temperatures, and pressures of the components. This is accomplished either using an analytical prescription or, more frequently, through tabulated EoS.

The system of equations (2)-(5) is a mixed hyperbolicparabolic system. All the terms on the right-hand side of the equations are operator-split from the solution of the nonideal single-fluid magneto-hydrodynamics. The latter is handled using the single-step, time marching algorithm of the unsplit staggered mesh (USM) ${ }^{40,41}$ for Cartesian coordinates and its extension to cylindrical systems. ${ }^{42}$ Both resistivity and the Biermann battery term are included in the staggered electric field, which allows us to preserve magnetic field solenoidality at machine accuracy through constrained transport.

In order to utilize $3 \mathrm{~T}$ EoS and properly distribute the update of $e_{\text {int }}$ to its components, we advance the auxiliary equations (6)-(8). However, the work terms $p_{s} \nabla \mathbf{u}$ (with $s$ denoting ions, electrons, or radiation) are ill-defined at shocks. To overcome this, we employ a method inspired by the radiation-hydrodynamics code RAGE, ${ }^{43}$ which distributes the change due to work and total shock-heating, recovered from the solution of equations (2)-(5), based on the pressure ratio of the components.

The right-hand side of equations (6)-(8) is in turn operator-split, and each physical process is handled separately. ${ }^{34}$ The physical processes represented in our formulation include energy exchange between ions and electrons through collisions, electron thermal conduction, and radiation transport in the multi-group, flux-limited diffusion approximation. The last two are solved implicitly using the HYPRE $^{44}$ library to retain large time steps. To model the laser heating, we utilize laser beams in the geometric optics approximation. These are comprised of rays whose paths are traced $^{45}$ through the computational domain, based on the local refractive index of each cell. The laser power is deposited at the inverse bremsstrahlung rate, which depends on local electron number density gradients and local electron temperature gradients. The HEDLP capabilities of FLASH have been recently exercised in a number of experiments, ${ }^{46-50}$ as well as in the experiments described in what follows.

\section{PREVIOUS EXPERIMENTS}

We have developed an experimental program to exploit the similarity in scaled laboratory experiments. In a first set of experiments done using the LULI2000 laser at the Laboratoire d'Utilisation des Lasers Intenses in France, we successfully demonstrated the creation of seed magnetic fields at asymmetric shocks ${ }^{51}$ by the Biermann battery effect, ${ }^{5}$ as predicted by protogalactic structure formation simulations. ${ }^{4}$ In these experiments, a carbon rod target was placed in a chamber filled with helium gas. Laser beams were focused on the target, vaporizing part of it and launching an asymmetric shock into the gas. The seed magnetic fields created at the shock by the Biermann battery effect were measured using three-axis induction coils.

In a second set of experiments done using the Vulcan laser at the Rutherford-Appleton Laboratory in the UK, we showed that seed magnetic fields can be amplified in plasmas by the turbulence produced as shocks interact with strong density inhomogeneities, ${ }^{48}$ reminiscent of what is observed in the supernova remnant Cassiopeia A. ${ }^{52}$ In these experiments, a carbon rod target was placed in a chamber filled with argon gas. Laser beams were again focused on the target, vaporizing part of it and launching an asymmetric shock into the gas. The interaction of a shock with large density perturbations was reproduced in the laboratory by passing 
the shock through a plastic mesh (see Fig. 1, panels a and b). In this case, the seed magnetic fields produced by the Biermann battery effect were amplified by the turbulence produced when the shock passed through the grid. The amplified magnetic field was measured using three-axis induction coils. Due to the relatively small electron temperatures, the plasma was characterized by large magnetic resistivity and magnetic Reynolds numbers $\mathrm{Rm} \sim 1$; as a result, the field was amplified due to tangling and the magnetic energy followed a $k^{-11 / 3}$ Golitsyn power law, a consequence of balancing field advection and resistive diffusion. ${ }^{15,53}$

In the third set of experiments done using the Vulcan laser at the Rutherford-Appleton Laboratory in the UK, we demonstrated the ability to achieve developed turbulence and the higher magnetic Reynolds numbers (i.e., the higher velocities and temperatures) needed to produce greater amplification of seed magnetic fields, a precursor to turbulent dynamo. ${ }^{49}$ In these experiments, lasers were focused on two foil targets in a chamber filled with argon gas, producing plasma jets that collided in the center (see Fig. 1, panels $\mathrm{c}$ and d). The collision of the two jets produced developed turbulence in the interaction region that amplified the seed magnetic fields created by the Biermann battery effect. However, the Rm values that were obtained $(\sim 10)$ were still small for dynamo action. ${ }^{15,54}$

Building on these results, we have conceived and designed an experimental platform for the Omega laser facility at the Laboratory for Laser Energetics at the University of Rochester, in order to demonstrate and study the turbulent dynamo mechanism. While the platform combines key elements of our previous experiments to generate turbulent plasmas and modest amplifications of seed magnetic fields, it differs in one crucial aspect: according to the simulations, it may be possible to reach high enough Rm values for turbulent dynamo to operate.

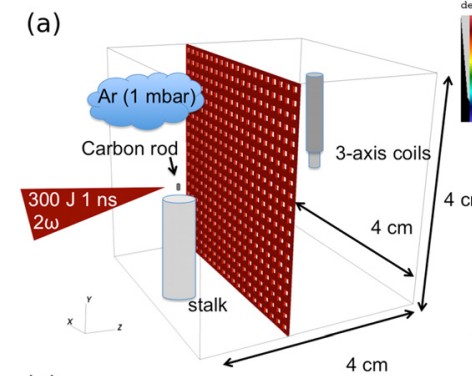

(c)

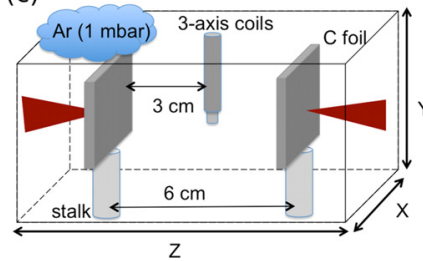

Z
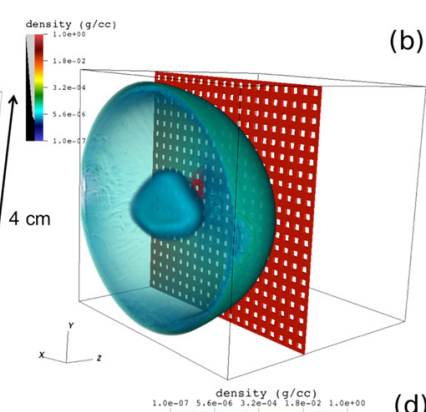

(b)

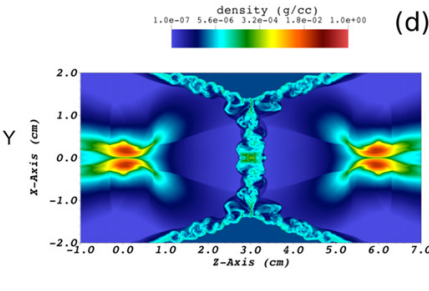

FIG. 1. Schematics and numerical simulations of previous experiments conducted at Vulcan. (a) Cartoon of the rod-grid experiment. ${ }^{48}$ (b) 3 D FLASH simulation of the rod-grid experiment. Displayed is the density logarithm when the shock traverses the plastic grid, stirring turbulence that amplifies the Biermann battery generated field by a factor of two. ${ }^{48}$ Numerical models of this experiment ${ }^{34}$ enabled the interpretation of the experimental results. (c) Cartoon of the colliding flows experiment, ${ }^{49}$ where higher Rm values where obtained. (d) 2D cylindrical FLASH simulation of the colliding flows experiment. $^{49}$

\section{DESIGN SIMULATIONS}

\section{A. Target design}

To design the experiments, we conducted an extensive series of 2D-cylindrical FLASH radiation-MHD simulations, followed by a smaller set of 3D FLASH radiation-MHD simulations on the Mira supercomputer at ANL. The simulations led to an experimental design that combines key elements of each of our two earlier experiments on Vulcan: ${ }^{48,49}$ a hot plasma flowing through a grid in the first and two plasma jets colliding in the second. The broad design goals consisted of obtaining

- a large kinetic energy reservoir in the turbulent flow to amplify the magnetic fields to measurable values;

- large magnetic Reynolds numbers, i.e., high temperatures and velocities, for the turbulent dynamo to operate; and

- sustained turbulence that would persist for a few eddy turnover timescales_at the driving scale-so as to amplify the field to saturation values.

In this design, the assembly is comprised of two composite targets and two grids that are connected by four boron rods (Figure 2(a)). The composite targets are $3 \mathrm{~mm}$ in diameter and consist of a chlorine-doped polystyrene foil, $50 \mu \mathrm{m}$ thick, and a polystyrene washer, $240 \mu \mathrm{m}$ thick (Figure 2(c)). The polystyrene washers were machined so as to have a 400 $\mu$ m-diameter cylindrical "well" in their centers. The two targets are mounted $8 \mathrm{~mm}$ apart (the distance measured from the proximate faces of the foils), and the pair of grids is placed between them. The two grids, made of polyimide, are

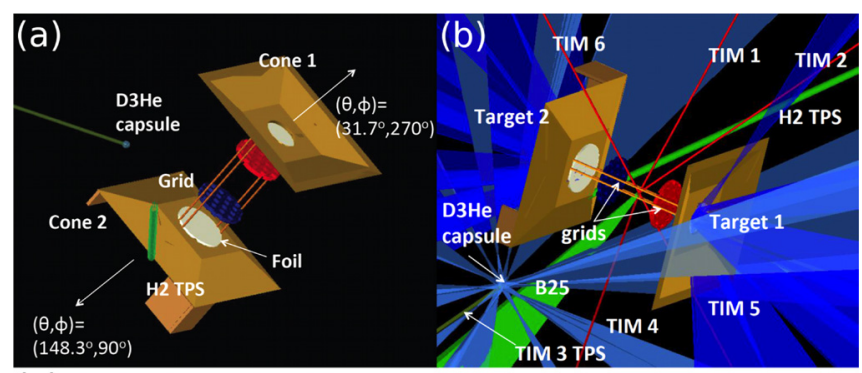

(c)

(d)

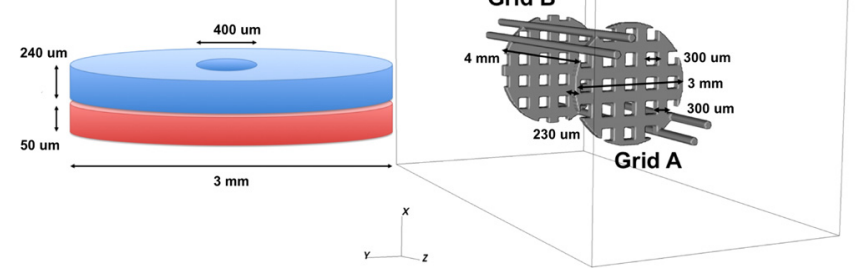

FIG. 2. (a) VisRad (http://www.prism-cs. com/Software/VisRad/VisRad.htm) target configuration, oriented in the Omega chamber. The two targets are placed opposite to each other. A pair of grids is situated in the propagation path of the flows, while two cones act as shields to both the interaction region and the diagnostic instruments. The small $\mathrm{D}^{3} \mathrm{He}$ capsule next to the assembly is the proton source for proton radiography. (b) VisRad experimental configuration. The blue beams show the drive on the two targets and the $\mathrm{D}^{3} \mathrm{He}$ capsule. The six TIMs (red lines) show the position of the diagnostics. (c) Detail of the composite targets: a polystyrene washer with a cylindrical "well" is placed on top of a thin chlorine-doped polystyrene foil. (d) Design specifications for the polyimide grids, through which the plasma flows will propagate. 
mounted $4 \mathrm{~mm}$ apart-the distance is once more measured with respect to their proximate faces-each of them $2 \mathrm{~mm}$ away from the respective proximate face of the foil-target. The grids have a diameter of $3 \mathrm{~mm}$ and a thickness of $230 \mu \mathrm{m}$. The opening fraction of each grid is $25 \%$, with 300 $\mu \mathrm{m}$-wide holes and a spacing of $300 \mu \mathrm{m}$ (Figure 2(d)). The hole patterns of the grids are offset by $300 \mu \mathrm{m}$ with respect to each other, thus breaking the mirror symmetry of the assembly: grid A has a hole in the center, while grid B does not. Rectangular cones on each target shield the diagnostics from the intense X-ray emission produced when a sequence of ten 1-ns duration laser beams coming from different angles illuminate each target (Figure 2(b)).

The two targets are driven for either 5 or $10 \mathrm{~ns}$, delivering a total of $5 \mathrm{~kJ}$ on an area defined by the laser phase plates. The radial profile of each beam's circular spot on the target can be approximated by a super-Gaussian of exponent 6 and an efolding radius of $336 \mu \mathrm{m}$; however, due to variation in the incidence angle, the illuminated area on each target is the overlap of ten ellipses. The temporal profile of the drive is either a 10 ns "top-hat" - each 1-ns long beam is fired sequentially so as to deliver $500 \mathrm{~J}$ per ns-or a "staircase" profile, ramping up the power towards the end of the drive $(500 \mathrm{~J} / \mathrm{ns}$ for $2 \mathrm{~ns}, 1000 \mathrm{~J} / \mathrm{ns}$ for $1 \mathrm{~ns}$, and $1500 \mathrm{~J} / \mathrm{ns}$ for $2 \mathrm{~ns}$ ).

The platform described above was designed based on our previous experiments and scores of 2D FLASH cylindrical simulations; many of its elements reflect the design goals stated at the beginning of this section. The machined washers act as collimators to direct the kinetic energy of the flows towards the collision region, minimizing lateral expansion; the offset of grids A and B results in corrugated fronts that will interleave, shear, and trigger Kelvin-Helmholtz instabilities that maximize mixing and the duration of turbulence; and the thickness of the foil components of the targets was selected so as to achieve large velocities while avoiding shine-through of the driving lasers, which could disrupt the turbulent flow and generate strong Biermann battery magnetic fields. ${ }^{55}$

While 2D simulations can provide useful information in the platform design process, they are not able to reproduce the experiment with high fidelity. MHD turbulence in two dimensions behaves differently than in three dimensions ${ }^{56}$ and, according to anti-dynamo theorems, ${ }^{57}$ cannot sustain dynamo. Moreover, the experimental platform has features that break the cylindrical symmetry assumed by our 2D modeling, which can have significant repercussions on the flow dynamics. Good examples are the square holes of the grids, the presence of the support rods, and asymmetries in the laser drive- a consequence of variance in directions and incidence angles of the laser beam sequences that irradiate the foils. To model the experiment properly, three dimensional simulations are required.

\section{B. Three-dimensional simulations}

In this section, we discuss the characteristics of four different 3D FLASH simulations that reflect the majority of the experimental configurations that we fielded at the Omega laser facility (see also Table I). The simulations vary in terms of the material properties of the targets (density and
TABLE I. Simulation key, target characteristics, and drive.

\begin{tabular}{lccc}
\hline \hline Simulation $^{\mathrm{a}}$ & Composition $^{\mathrm{b}}$ & Density $^{\mathrm{c}}$ & Drive \\
\hline $1 \mathrm{Cl} 10 \mathrm{~ns}$ & $\mathrm{C}(50.4 \%) \mathrm{H}(48.3 \%) \mathrm{Cl}(1 \%)$ & $1.29 \mathrm{~g} \mathrm{~cm}^{-3}$ & $10 \mathrm{~ns}$ \\
$6 \mathrm{Cl} 10 \mathrm{~ns}$ & $\mathrm{C}(49.9 \%) \mathrm{H}(43.8 \%) \mathrm{Cl}(6 \%)$ & $1.55 \mathrm{~g} \mathrm{~cm}^{-3}$ & $10 \mathrm{~ns}$ \\
$1 \mathrm{Cl} 5 \mathrm{~ns}$ & $\mathrm{C}(50.4 \%) \mathrm{H}(48.3 \%) \mathrm{Cl}(1 \%)$ & $1.29 \mathrm{~g} \mathrm{~cm}^{-3}$ & $5 \mathrm{~ns}$ \\
$6 \mathrm{Cl} 15 \mathrm{~ns}$ & $\mathrm{C}(49.9 \%) \mathrm{H}(43.8 \%) \mathrm{Cl}(6 \%)$ & $1.55 \mathrm{~g} \mathrm{~cm}^{-3}$ & $5 \mathrm{~ns}$ \\
\hline \hline
\end{tabular}

${ }^{\mathrm{a}}$ The key for each simulation is defined by the chlorine doping percentage and the drive duration.

${ }^{\mathrm{b}}$ The composition refers only to that of the foil part of the target. The composition of the washer is that of regular polystyrene $(\mathrm{CH})$.

${ }^{\mathrm{c}}$ In comparison, the density of the washer is $1.07 \mathrm{~g} \mathrm{~cm}^{-3}$, which highlights the density increase due to the chlorine doping.

composition of the foils) and the shape and duration of the laser drive. The initial conditions reflect the design specifications of the platform, discussed in Sec. IV A. In a computational domain that spans $0.625 \mathrm{~cm}$ in $X$ and $Y$ and $1.250 \mathrm{~cm}$ in $Z$, we initialize the targets, grids, and rods that we described in Figure 2, at a temperature of 290 K. A snapshot of the initial condition for case 1Cl10ns (logarithm of electron number density and contours of the grids and rods) is shown in Figure 3(a). To simplify the initialization, we omit the diagnostic shields and extend our targets to the domain boundaries, effectively separating the back of the foils-where laser illumination occurs-from the domain center.

The domain is resolved with $\sim 3.3 \times 10^{7}$ cells, corresponding to $\sim 25 \mu \mathrm{m}$ per cell width. The boundary conditions on all sides of the computational box are set to "outflow" (zero-gradient), except for the normal component of the magnetic field, which is recovered through the solenoidality condition. For the multigroup flux-limited radiation diffusion, we consider 6 energy bins from $0.1 \mathrm{eV}$ to $100 \mathrm{keV}$. To model accurately the material properties of the chlorinated targets, we utilize opacity and EoS tables computed with PROPACEOS. ${ }^{58}$ Temporal integration of the non-ideal 3T MHD equations is carried out for $50 \mathrm{~ns}$, using the second-order unsplit time-marching method of the USM algorithm, ${ }^{41}$ an extension of the corner transport upwind (CTU) approach. ${ }^{59}$ Spatial reconstruction is done utilizing the piecewise parabolic method ${ }^{60}$ (PPM) and a minmod limiter. The upwind fluxes are computed with a Harten-Laxvan Leer Contact ${ }^{61}$ (HLLC) Riemann solver. Implicit solvers for radiation and electron thermal conduction are carried out using a conjugate gradient method (PCG), preconditioned with algebraic multigrid (AMG), as implemented in the HYPRE library.

To model accurately the laser drive, we implemented the spatial and temporal specifications of each of the twenty Omega driver beams separately. This was done to ensure that the interplay between obliqueness of incidence angle and target deformation due to the drive would be captured correctly. Each $3 \omega$ beam is simulated using 16,000 rays per timestep, achieving good statistics and low Poisson noise in the energy deposition. The $5 \times 10^{11} \mathrm{~W}$ power in each beam is distributed assuming the spatial beam profile, mentioned above. 


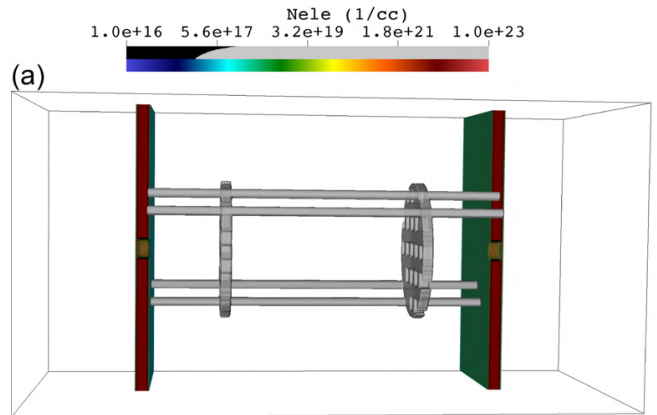

(b)

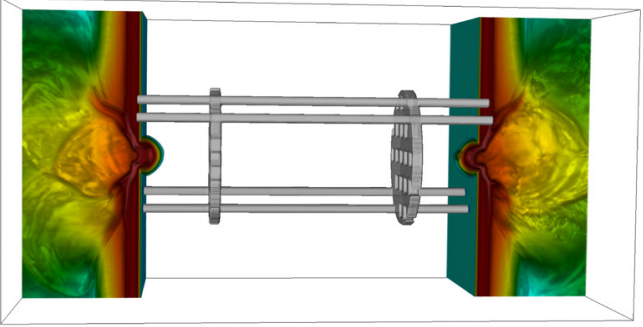

(c)

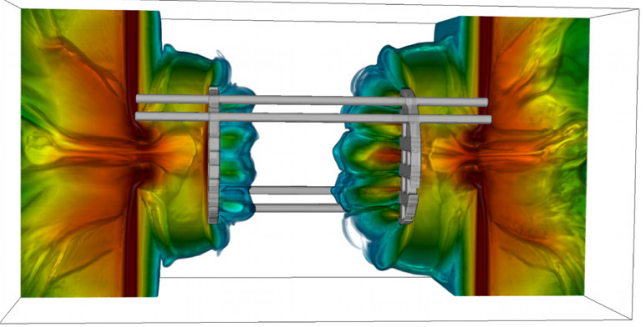

(d)

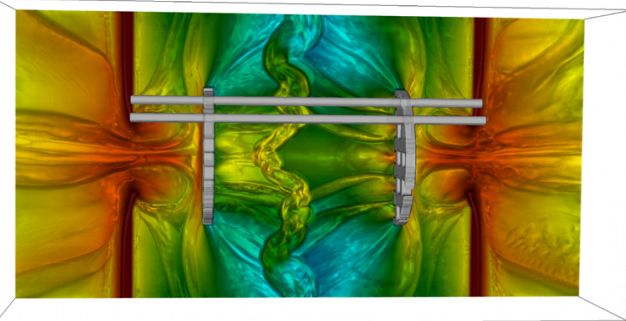

(e)

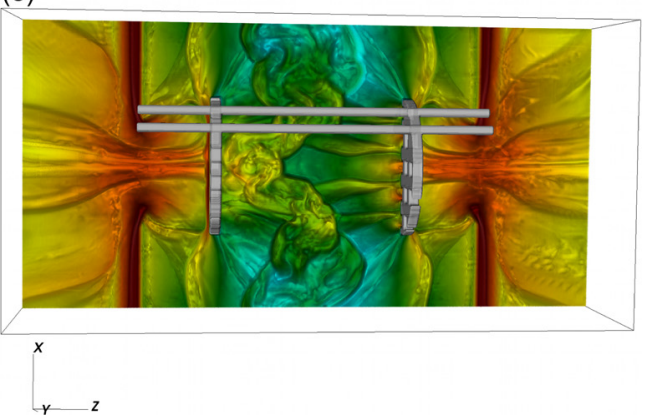

FIG. 3. Initial condition and temporal evolution for the 1Cl10ns simulation. (a) Electron number density logarithm (half-rendering) and contours of the grids and supporting boron rods at $t=0 \mathrm{~ns}$. (b) Same as (a) but for $t=8 \mathrm{~ns}$. (c) Same as (a) but for $t=20 \mathrm{~ns}$. (d) Same as (a) but for $t=35 \mathrm{~ns}$. (e) Same as (a) but for $t=45 \mathrm{~ns}$.

The temporal evolution of the system is shown in Figure 3. In the simulations, the laser beams ablate the back of the foil targets and a pair of hot plasma plumes is created and expands outwards. The laser-target interaction generates strong magnetic fields due to the Biermann battery mechanism ${ }^{55}$ which are "flux-frozen" into and advected by the plasma. The ablation results in a pair of shocks-driven inside the chlorinated polystyrene foils- that break out and propagate supersonically towards the grids (Figure 3(b)). The lateral expansion of the inwards-moving plasma flows is inhibited by the collimating effect of the washers. The laser drive (for this case) persists for $10 \mathrm{~ns}$ and is turned off shortly after the break-out. Subsequently, the flows traverse the grids to form "finger" formations and corrugated fronts of a characteristic length-scale $\mathcal{L} \sim 600 \mu \mathrm{m}$-the sum of a hole width and a hole spacing - and continue towards the center of the domain (Figure 3(c)). The flows then collide to form a cupshaped interaction region of hot, subsonic turbulent plasma with an outer scale defined by $\mathcal{L}$ (Figure 3(d)). The bottom of the "cup" is pointing towards grid $\mathrm{B}$, a result of $\mathcal{L}$ being comparable to the thickness of the interaction region: as grid A has a center hole, the locally increased mass flux from grid A results in the deformation. At late times (Figure 3(e)), the interaction region thickens and slowly drifts towards grid B, gradually cooling by advection (primarily) and radiation.

\section{DISCUSSION}

\section{A. Predicted plasma properties}

The general behavior described in Figure 3 occurs in all four simulations, but the change in the composition and drive affects both the timing of events and the plasma properties. This becomes apparent when comparing the front positions at the same evolution time. In Figure 4, we display a halfrendering of the electron density logarithm at $20 \mathrm{~ns}$ for all cases; the 6\% chlorine-doped cases (panels a and b) appear slower than the $1 \%$ cases (panels $\mathrm{c}$ and d). This is a consequence of variation in the foil target density, which is $20 \%$ larger for cases $6 \mathrm{Cl} 10 \mathrm{~ns}$ and $6 \mathrm{Cl} 5 \mathrm{~ns}$. Similarly, the decrease in the drive duration from 10 to $5 \mathrm{~ns}$ directly translates to an increase of laser intensity, which results in faster flows for cases $6 \mathrm{C} 5 \mathrm{~ns}$ and $1 \mathrm{Cl} 5 \mathrm{~ns}$ (panels b and d) than their $10 \mathrm{~ns}$ counterparts (panels a and c). In all cases, however, the flows eventually collide to form a turbulent interaction region, reaching high temperatures that endure for several nanoseconds. Thus, a natural separation in terms of analyzing the simulation results is to consider the flows prior to collision and

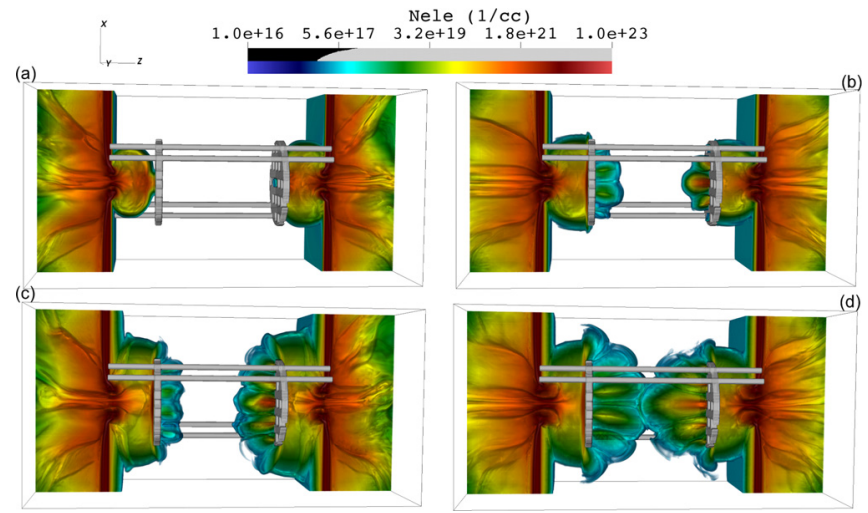

FIG. 4. Front positions (electron density logarithm) at $20 \mathrm{~ns}$ for the various simulated cases: (a) 6Cl10ns, (b) 6Cl5ns, (c) $1 \mathrm{Cl} 10 \mathrm{~ns}$, and (d) $1 \mathrm{Cl} 5 \mathrm{~ns}$. 
the turbulent region after the collision, circumventing the temporal offsets due to different drives and compositions.

To present quantitative results, we utilize a control volume, a cubic box of an edge length of $500 \mu \mathrm{m}$, to sample relevant plasma quantities before and after collision. In the former case, the box tracks in time the propagating plasma front from grid A, centered at the edge of the front. Postcollision, the tracking volume is pinned in the interaction region, centered at the stagnation point formed by the colliding fronts. The box is allowed to move along the line of centers (LoC) that is parallel to the $Z$ axis and intersects the centers of the targets $(X=Y=0)$. A comprehensive list of plasma properties for case 1Cl10ns is given in Table II. Similar values are also recovered for the remaining cases with some variation due to the drive and composition difference.

The plasma remains highly collisional throughout the simulation, and the MHD treatment is valid; the distribution function can be approximated with a Maxwellian. ${ }^{30}$ Prior to collision, the flows are mildly supersonic $(M \sim 2-3)$. As the plasma flows traverse the grids, weak shocks are formed that result in the heating of ions, whereas electron temperature lags slightly behind due to the initially long ion-electron equilibration timescale. Typical flow densities and temperatures are of the order of $\sim 10^{17}-10^{18} \mathrm{~cm}^{-3}$ and few tens of $\mathrm{eV}$, respectively. The flows propagate with velocities of a couple of hundred $\mathrm{km} \mathrm{s}^{-1}$ to meet at the domain center. From the laser-target interaction, we have the generation of strong Biermann battery ${ }^{5}$ magnetic fields, which are of the order of $\sim$ MG close to the targets and are advected with the plasma. The misaligned gradients of electron pressure and density continuously generate fields as the flows propagate, but advection causes substantial spatial dilution, reducing the field strength of the fronts down to values of $\sim 1-10 \mathrm{kG}$ prior to collision (Figure 6(a)).

The collision takes place at $\sim 24-25 \mathrm{~ns}$ for the $1 \mathrm{Cl} 10 \mathrm{~ns}$ case and results in a pair of accretion shocks with a subsonic turbulent region in between. The ion and electron temperatures increase to a few hundreds of eV (Figure 5(a)) and equilibrate rapidly. While such turbulent flows were recreated also in our simulations of the colliding jet experiment ${ }^{49}$ with the Vulcan laser, in the simulations of the Omega platform, we reach values of $\mathrm{Rm}$ in the many hundreds (Figure 5(b)). The high Rm values persist for several ns after the collision (Figure 5(b)), and the magnetic fields appear significantly amplified to peak values of hundreds of $\mathrm{kG}$ (Figure 6(b)).

In the simulations, the turbulent plasma is characterized by an outer scale $\mathcal{L} \sim 600 \mu \mathrm{m}$ and has a Kolmogorov-like spectrum (Figure 7). The dissipation scales are below our spatial resolution, both for viscous $\left(l_{\nu}=\mathcal{L} / \mathrm{Re}^{3 / 4} \sim 1 \mu \mathrm{m}\right)$ and resistive $\left(l_{\eta}=\mathcal{L} / \mathrm{Rm}^{3 / 4} \sim 4 \mu \mathrm{m}\right.$, for $\left.\mathrm{Pm}<1\right)$ dissipation. As a result, the simulations cannot capture the complete energy cascade but can inform us on the behavior of the energy spectra at larger scales - in the limited range allowed by our numerical resolution. Using the control volume mentioned above, we can recover the temporal evolution of the magnetic field strength and compute, at different times, the angle-integrated spectra of the magnetic and kinetic energy. The simulated time history of the field (peak values $B_{\max }$ and root mean square values $B_{\text {rms }}$ in the control volume) for case $1 \mathrm{Cl10ns}$ is given in Figure 7(a). This semi-log plot shows the sequence of events: initially $(t<24 \mathrm{~ns})$, the magnetic field decreases as the plasma expands (a dilution phase) to values of a few $\mathrm{kG}$, which will act as seed fields

TABLE II. Simulated plasma properties for case 1Cl10ns prior to and after collision.

\begin{tabular}{|c|c|c|c|}
\hline Plasma property & Formula & Prior to collision $^{\mathrm{a}}$ & After collision ${ }^{\mathrm{b}}$ \\
\hline Electron density $N_{\text {ele }}\left(\mathrm{cm}^{-3}\right)$ & $\ldots$ & $\sim 1 \times 10^{18}$ & $\sim 8 \times 10^{19}$ \\
\hline Ion density $N_{\text {ion }}\left(\mathrm{cm}^{-3}\right)$ & $\ldots$ & $\sim 4 \times 10^{17}$ & $\sim 3 \times 10^{19}$ \\
\hline Electron temperature $T_{\text {ele }}(\mathrm{eV})$ & $\ldots$ & $\sim 60-90$ & $\sim 150-350$ \\
\hline Ion temperature $T_{\text {ion }}(\mathrm{eV})$ & $\ldots$ & $\sim 100-120$ & $\sim 150-350$ \\
\hline Average ionization $Z$ & $\ldots$ & $\sim 3.6$ & $\sim 3.6$ \\
\hline Average atomic weight $A$ (a.m.u.) & $\ldots$ & $\sim 6.8$ & $\sim 6.8$ \\
\hline Flow velocity $u\left(\mathrm{~cm} \mathrm{~s}^{-1}\right)$ & $\cdots$ & $\sim 2 . \times 10^{7}$ & $\sim 1.4 \times 10^{7}$ \\
\hline Coulomb logarithm $\ln \Lambda$ & $23.5-\ln \left(N_{\mathrm{ele}}^{1 / 2} T_{\mathrm{ele}}^{-5 / 4}\right)-\sqrt{10^{-5}+\frac{\left(\ln \left(T_{\mathrm{ele}}\right)-2\right)^{2}}{16}}$ & $\sim 7.4-7.8$ & $\sim 6.1-6.9$ \\
\hline Sound speed $C_{\mathrm{s}}\left(\mathrm{cm} \mathrm{s}^{-1}\right)$ & $9.80 \times 10^{5} \frac{\left[Z T_{\text {ele }}+(5 / 3) T_{\text {ion }}\right]^{1 / 2}}{A^{1 / 2}}$ & $\sim 7-9 \times 10^{6}$ & $\sim 1.1-1.6 \times 10^{7}$ \\
\hline Mach number $M$ & $u / C_{\mathrm{s}}$ & $\sim 2-3$ & $\lesssim 1$ \\
\hline Ion-ion mean free path $\lambda_{i i}(\mathrm{~cm})$ & $2.88 \times 10^{13} \frac{T_{\text {ion }}^{2}}{Z^{4} N_{\text {ion }} \ln \Lambda}$ & $\sim 5-8 \times 10^{-4}$ & $\sim 0.2-1 \times 10^{-4}$ \\
\hline Magnetic Reynolds number Rm & $u \mathcal{L} / \eta\left(\eta=3.2 \times 10^{5} \mathrm{~cm}^{2} \mathrm{~s}^{-1} \frac{Z \ln \Lambda}{T_{\mathrm{ele}}^{3 / 2}}\right)$ & $\sim 60-120$ & $\sim 300-900^{\mathrm{c}}$ \\
\hline Reynolds number Re & $u \mathcal{L} / \nu\left(\nu=1.92 \times 10^{19} \mathrm{~cm}^{2} \mathrm{~s}^{-1} \frac{T_{\text {ion }}^{5 / 2}}{A^{1 / 2} Z^{4} N_{\text {ion }} \ln \Lambda}\right)$ & $\sim 540-850$ & $\sim 1300-8300$ \\
\hline Magnetic Prandtl number Pm & $\operatorname{Rm} / \operatorname{Re}$ & $\gtrsim 0.1$ & $\gtrsim 0.1$ \\
\hline
\end{tabular}

${ }^{a}$ Estimated in the tracking control volume, 2 ns prior to collision.

${ }^{\mathrm{b}}$ Characteristic values in a $10 \mathrm{~ns}$ time range after collision.

${ }^{\mathrm{c}}$ Peak values of 1300-1600 within the first 4 ns after collision, consistent with threshold estimates for the small Pm regime. ${ }^{15}$ 
(a)
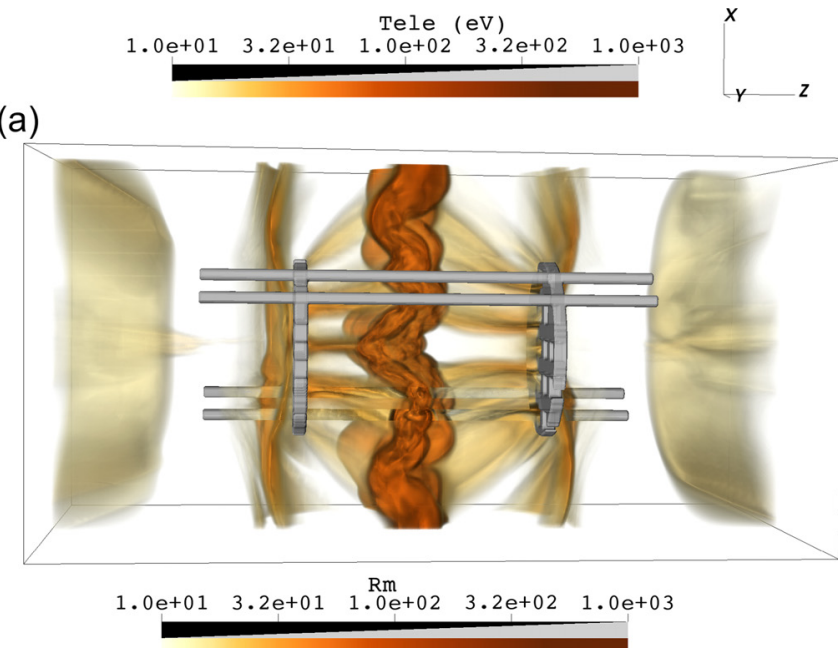

(b)

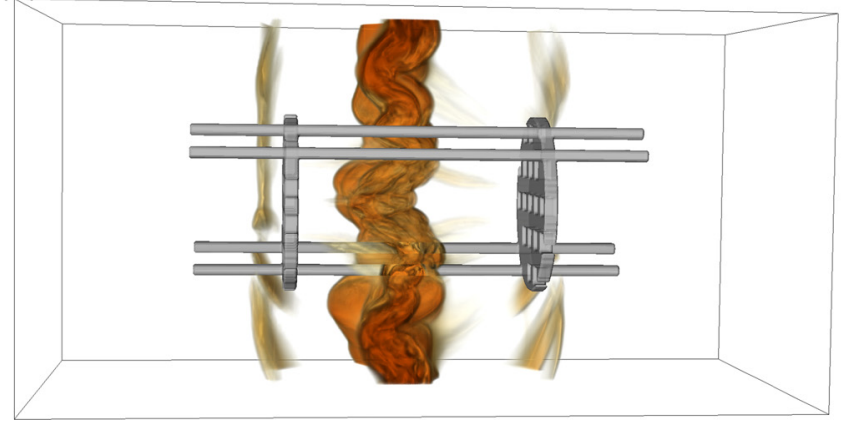

FIG. 5. Volume rendering of (a) the electron temperature in $\mathrm{eV}$ and (b) the magnetic Reynolds number (at scale $\mathcal{L}$ ), for case $1 \mathrm{Cl} 10 \mathrm{~ns}$ at $35 \mathrm{~ns}$.

(a)
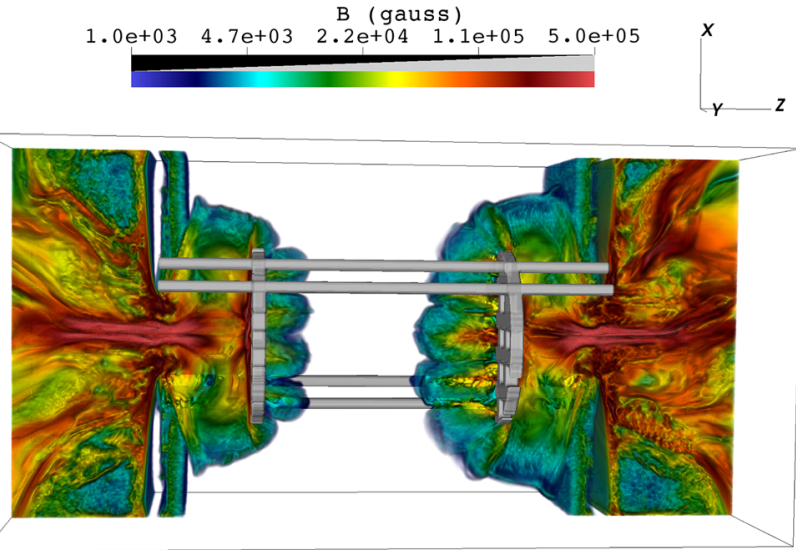

(b)

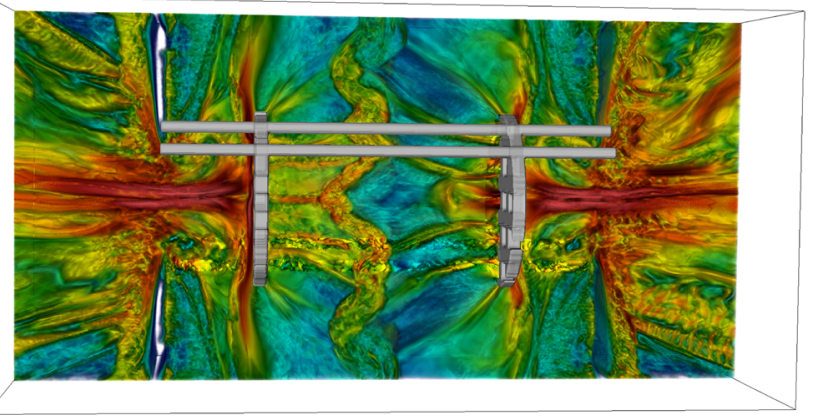

FIG. 6. Volume rendering of the magnetic field magnitude in gauss for the $1 \mathrm{Cl10 \textrm {ns }}$ case at (a) $20 \mathrm{~ns}$ and (b) $35 \mathrm{~ns}$. for the dynamo amplification; then collision occurs $(t \sim 24-25 \mathrm{~ns})$ and we see a sharp increase due to compression effects and an exponential increase phase $(t \sim 25-28$ ns) consistent with kinematic dynamo; subsequently $(t>28$ ns), the exponential growth phase ends as the field strength increases, entering a non-linear dynamo phase where the field becomes important with respect to the flow dynam$\mathrm{ics}^{62}$ the curve flattens at later times when saturation is reached, with peaks as high as $\sim 300-350 \mathrm{kG}$. This occurs on timescales that are comparable to an eddy turnover time at the outer scale, $t_{\mathcal{L}} \sim \mathcal{L} / u \sim 4$ ns. Figures 7 (b) and 7(c) show the simulated spectra for the one-dimensional, angleintegrated kinetic and magnetic energies

$$
E_{k}(k)=\frac{1}{2}\langle\rho\rangle \int d \Omega_{k} k^{2}\left\langle|\mathbf{u}(\mathbf{k})|^{2}\right\rangle \quad \text { and }
$$

$$
E_{m}(k)=\frac{1}{2} \int d \Omega_{k} k^{2}\left\langle|\mathbf{B}(\mathbf{k})|^{2}\right\rangle
$$

at different times-shortly after collision in panel $\mathrm{b}$ and at saturation in panel c. The kinetic energy follows approximately a $k^{-5 / 3}$ Kolmogorov power law, consistent with a subsonic turbulent plasma. The magnetic energy, on the other hand, follows a $k^{-1}$ power law, previously found for galactic turbulence ${ }^{63}$ and fluctuation dynamo at small magnetic Prandtl numbers. ${ }^{15}$ Shortly after collision (Figure 7(b)), the magnetic energy is considerably smaller than the kinetic energy. At saturation (Figure 7(b)), the magnetic energy rises up to $1-10 \%$ of the kinetic energy, depending on scale. Such saturation values were also recovered by other numerical studies of turbulent dynamo. ${ }^{54,64}$

As a whole, our numerical results suggest that the Omega laser experiments that we have simulated would be able to reach Rm close to critical values ${ }^{15}$ and thus enter the turbulent dynamo regime, to enable an experimental study of the properties of MHD turbulence and magnetic field amplification. For a discussion on the experimental findings, the reader is referred to in Ref. 30.

\section{B. Validation of the simulations}

During the experiment, we fielded a number of diagnostics to probe the plasma and magnetic field properties. ${ }^{30} \mathrm{~A}$ small subset of the experimental data can be used to validate specific properties of the simulations, such as the propagation speed of the colliding flows and the time of collision. For these, we utilize information from soft X-ray imaging and the Thomson scattering diagnostic. ${ }^{65}$

Experimental X-ray images taken at early times of the evolution allow us to track the position of the plasma fronts prior to collision. These are given in Figures 8(a) and 8(c). The experimental configurations correspond to our $6 \mathrm{Cl} 10 \mathrm{~ns}$ case at 24 and $31 \mathrm{~ns}$, respectively. Notice that in the case of panel a, we only drove the target on the side of grid A and grid B was missing from the assembly. This can be reproduced with our $6 \mathrm{Cl} 10 \mathrm{~ns}$ simulation by omitting the evolution from the side of grid B. The agreement between experimental data and simulation results is fairly good (Figures 8(b) and $8(\mathrm{~d})$ ). The heavily chlorinated target propagates 

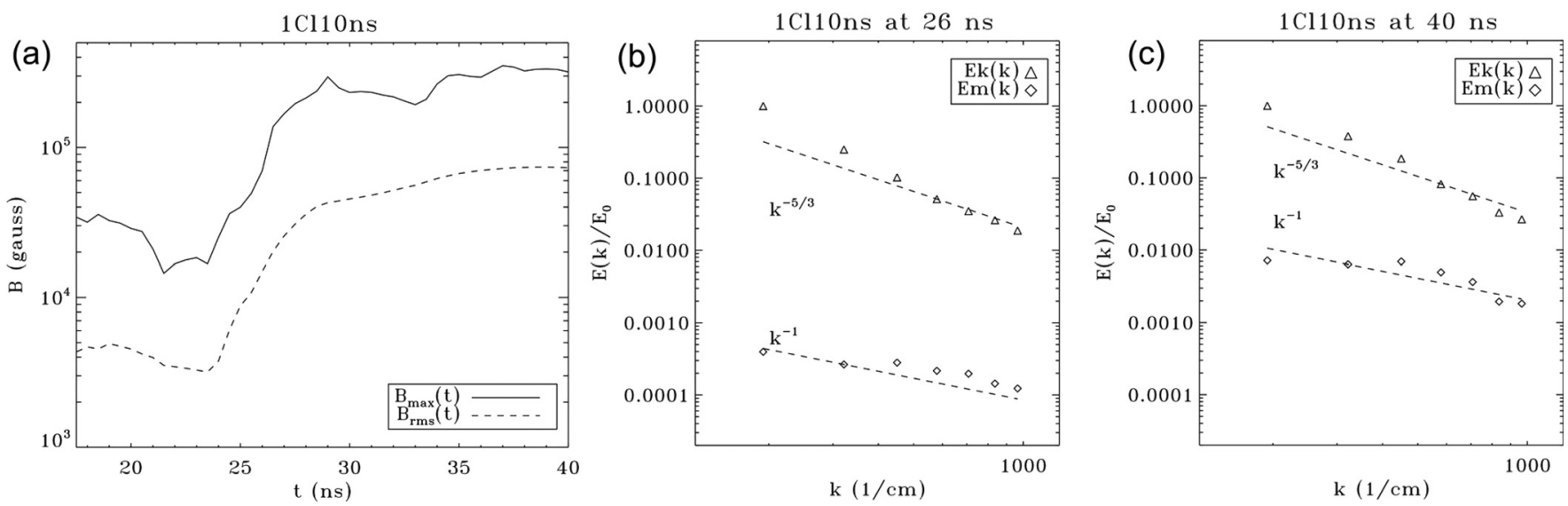

FIG. 7. (a) Magnetic field strength (maximum value and root mean square value) as a function of time in the tracking control volume. (b) Angle integrated kinetic $\left(E_{k}\right)$ and magnetic energy $\left(E_{m}\right)$ as a function of $\mathrm{k}$, for case $1 \mathrm{Cl1}$ ns at $\mathrm{t}=26 \mathrm{~ns}$. The values are normalized to the kinetic energy at the largest scale, and we display a scale range between 50 and $500 \mu \mathrm{m}$. Immediately after collision, the magnetic energy is only a small fraction of the kinetic energy. The magnetic energy follows a power law consistent with $\mathrm{k}^{-1}$, while the kinetic energy displays a Kolmogorov slope. At the largest scales, there is a steepening due to bulk motion in the $\mathrm{Z}$ direction. (c) Same as (b) but for $\mathrm{t}=40 \mathrm{~ns}$. At saturation, the magnetic energy becomes comparable to the kinetic energy (1\%-10\%). The kinetic energy slope-steepening at large scales is less pronounced as the turbulence homogenizes more.

slowly-slower than the rest; see also Figure 4-and at 24 ns the front has just crossed grid A. At $31 \mathrm{~ns}$, the two flows are clearly visible and yet to meet at the center of the chamber. It should be noted that the X-ray emission depends on the plasma density; therefore panel c only shows emission from the denser parts of the flow. The image is unfortunately saturated due to diagnostic filter options, and we cannot discern variations in the plasma structure.

To bound the collision timing and validate the numerically predicted time, we can utilize information from the Thomson scattering diagnostic. The spectrum of light produced by Thomson scattering in a hot plasma, in particular the shape and position of the ion feature that results from collective processes involving excitation of ion-acoustic wave modes, depends sensitively on the plasma velocity, electron density, electron temperature, and ion temperature. A scattering diagnostic based on this effect thus allows detailed inferences of these physical quantities. ${ }^{65} \mathrm{~A} 2 \omega$ low energy beam is focused on a small spot in the plasma; a dedicated detector records the radiation from a narrow angle range to produce a time-streaked image of the scattered light from the small target volume. In one of the shots, corresponding to our $1 \mathrm{Cl10ns}$ case, the diagnostic probed the interaction region in the time interval 24.5-27.5 ns, which overlaps with the numerically predicted collision time ( 24-25 ns). While a typical spectrum would exhibit only one pair of ion features (two peaks in the intensity profile), in this case, we observed four peaks (Figure 9(a)). This occurs when the light scatters off counter-streaming plasma, i.e., when the plasma fronts converge. At later times, the four peaks merge into two, an indication that the interaction region has formed in the experiment. This sequence and timing of events matches fairly closely the simulation results, within $\sim 1-2$ ns (Figure 9(b) and 9(c)). It should be noted that this agreement was achieved without tuning of the laser energy deposition, which can sometimes be necessary to account for laser-plasma interaction (LPI) effects that can reduce the drive efficiency.

\section{Comparison of simulated diagnostics and experimental data}

There has been considerable effort in the FLASH code development to implement synthetic counterparts of (a)

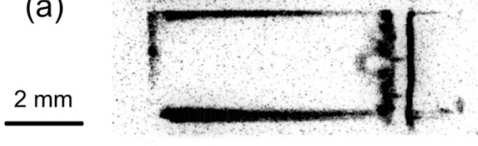

(b)
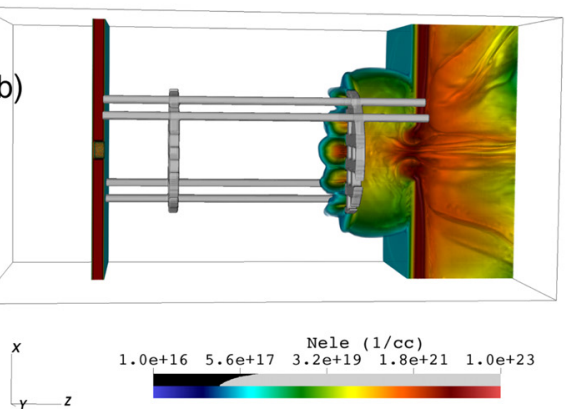

(c)
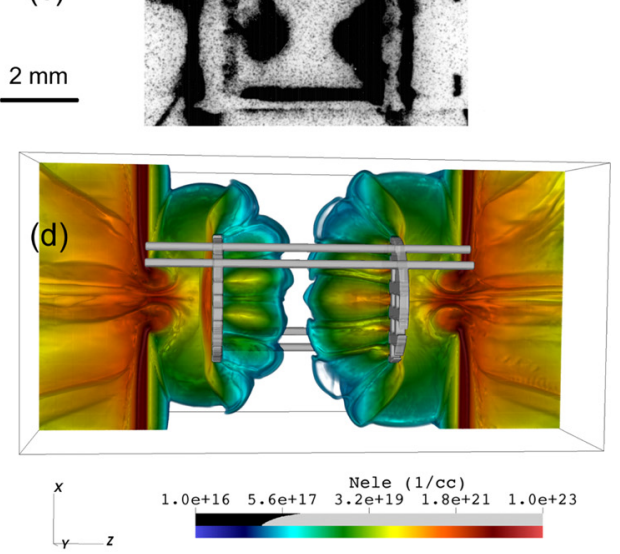

FIG. 8. Soft X-ray images of the plasma fronts at two different times: (a) $24 \mathrm{~ns}$ and (c) $31 \mathrm{~ns}$. The experimental configuration corresponds to our simulation case 6Cl10ns. (b) Rendering of the electron density logarithm for our $6 \mathrm{Cl} 10 \mathrm{~ns}$ simulation at $24 \mathrm{~ns}$, omitting the plasma from grid B. (d) Rendering of the electron density logarithm for our $6 \mathrm{Cl1}$ ns simulation at $31 \mathrm{~ns}$. 
(a)

(b)

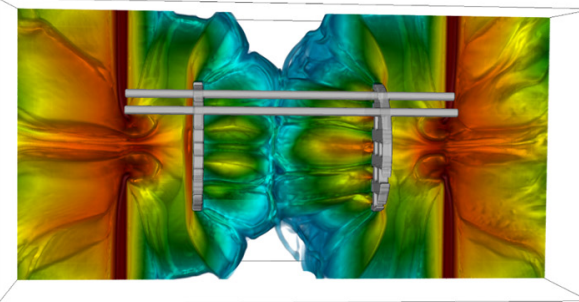

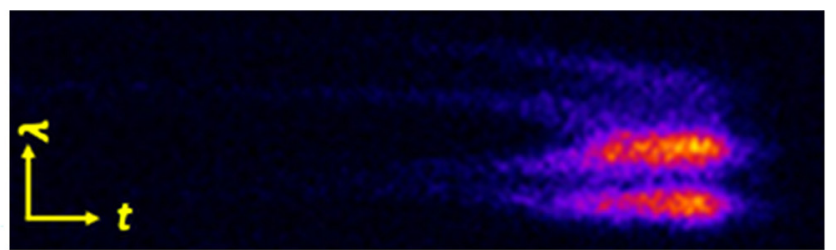

(c)

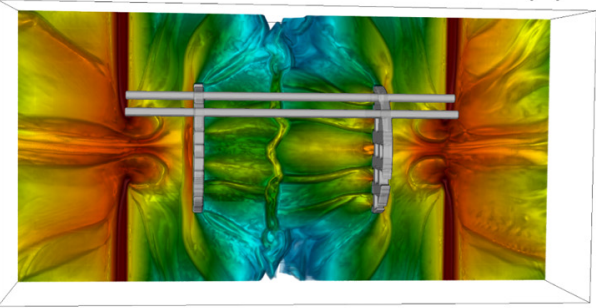

$\mathrm{t}=24.5 \mathrm{~ns}$ $\mathrm{t}=27.5 \mathrm{~ns}$

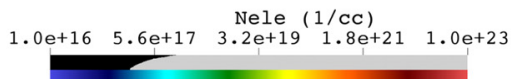

FIG. 9. Flow collision timing from the Thomson scattering diagnostic for an experimental shot that corresponds to our $1 \mathrm{Cl} 10 \mathrm{~ns}$ case. (a) Time-streaked image of the Thomson scattered light, with a temporal resolution of $\sim 50 \mathrm{ps}$. The four peaks correspond to a pair of ion features that move in opposite directions and merge at late times as a single-flow plasma forms. The image corresponds to the 24.5-27.5 ns time interval, indicating that collision occurs between 25-26 ns. (b) Volume rendering of the electron number density logarithm at $24.5 \mathrm{~ns}$ for case $1 \mathrm{Cl10ns}$. The counter-streaming flows reach the probing region. (c) Same as (b) at $27.5 \mathrm{~ns}$. The turbulent interaction region is well-formed. experimental diagnostics that are commonly used by the HEDLP community. This reflects an effort to cast simulation results in a format that allows them to be compared directly to the data, minimizing post-processing of the latter and including many of the physical and statistical processes that go into the creation of the experimental image. Here, we consider three of the diagnostics that were fielded in our Omega experiment, ${ }^{30}$ used to probe the state of the plasma and the magnetic field in the interaction region.

$\mathrm{X}$-ray imaging. The X-ray imaging, which was used above to validate the FLASH predictions of the propagation speed of the plasma fronts, can provide useful information regarding the shape and properties of the interaction region. To create synthetic images from the FLASH simulations, the results were recast to be read in post-processing by SPECT3d, ${ }^{67}$ a collisional-radiative spectral analysis code designed to simulate atomic and radiative properties of laboratory plasmas. We create synthetic images for case $1 \mathrm{Cl} 5 \mathrm{~ns}$ at 27 and $31 \mathrm{~ns}$ and compare them with the experimentally recovered X-ray images (Figure 10). In the experimental results, we can see that the interaction region is well-formed by $27 \mathrm{~ns}$ (panel a), with the characteristic "cup" shape predicted by our numerical models (we remind the reader that case $1 \mathrm{Cl} 5 \mathrm{~ns}$ was the one that exhibited flow collision very early on at $\sim 20-21 \mathrm{~ns}$, see also Figure 4 ). At $31 \mathrm{~ns}$, the interaction region has become slightly thicker (panel b). The FLASH/SPECT3d synthetic images are in good agreement with the overall shape and distinct features of the interaction region (panels $\mathrm{c}$ and $\mathrm{d}$ ), exhibiting also the same trend in the thickness. The turbulence also has a measurable effect on $\mathrm{X}$-ray emissivity. The 2D fluctuations in X-ray intensity can be related to the $3 \mathrm{D}$ density fluctuations ${ }^{66}$ and, under specific caveats which include negligible electron temperature fluctuations and isotropic turbulence, have proportional power laws. A formal discussion on this proportionality and on $\mathrm{X}$-ray image analysis is presented in Ref. 30. The spectral analysis for the $31 \mathrm{~ns}$ experimental image is consistent with a 3D Kolmogorov power law $k^{-11 / 3}$ (Figure 10(e)); this corresponds to a $1 \mathrm{D}$ power spectrum $\propto k^{2} k^{-11 / 3} \sim k^{-5 / 3}$. If the caveats apply and the interaction region in the experiment is indeed subsonic, as the simulations seem to indicate, then the density would behave as a passive scalar and the kinetic energy power spectrum would also follow a $k^{-5 / 3}$ power law. Such a result would agree with the FLASH prediction (Figure 7(b) and 7(c)).

Thomson scattering. As we mentioned above, the Thomson scattering diagnostic probes the plasma with a low-energy $2 \omega$ laser beam $(526.5 \mathrm{~nm})$ to produce a light (a)

(c)

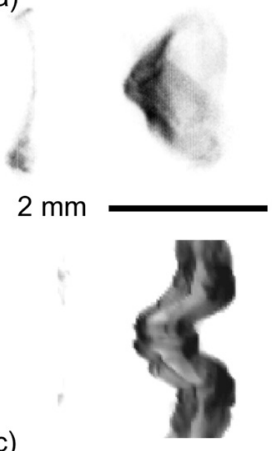

(b)

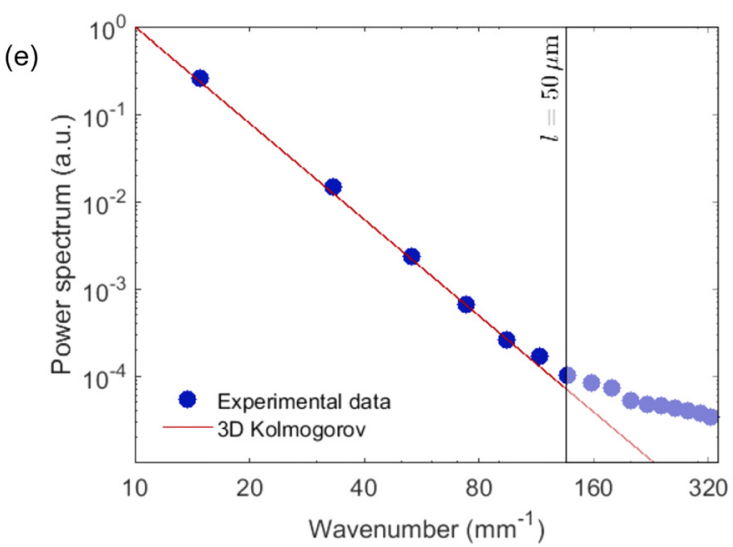

FIG. 10. Experimental and synthetic X-ray images and power spectrum. (a) Soft X-ray experimental image at $27 \mathrm{~ns}$ for a shot corresponding to our $1 \mathrm{Cl} 15 \mathrm{~ns}$ case. (b) Same as (a) but at $31 \mathrm{~ns}$. (c) Synthetic X-ray image from the FLASH results for case $1 \mathrm{Cl} 5 \mathrm{~ns}$ at $27 \mathrm{~ns}$. (d) Same as (c) but at $31 \mathrm{~ns}$. (e) Power spectrum recovered from the spectral analysis of the interaction region in panel (b). The power spectrum of $2 \mathrm{D}$ intensity fluctuations is proportional to the $3 \mathrm{D}$ spectrum of density fluctuations ${ }^{30,66}$ and is consistent with Kolmogorov turbulence. The deviation seen in small scales is attributed to Poisson noise. 
spectrum with features sensitive to the plasma characteristics. ${ }^{65}$ We have implemented in FLASH a simulated Thomson scattering diagnostic to reproduce such spectra. The code module computes multiple ray paths, each going from a lens location to a scattering location and then from the scattering area to the detector (alongside the diagnostic rays, we also launch rays from our laser package to account for any laser heating effects ${ }^{30}$ ). While the lens center and the detector location are held fixed, multiple ray paths are generated by iteration over points in the part of the region of interest, at subcell resolution. We perform an integration along the paths to compute the attenuation of ray power by inverse bremsstrahlung; the simulated diagnostic thus takes into consideration the effect of matter present in the chamber on both the incoming and the scattered light via absorption. Each ray determines a scattering angle and plasma state (electron density, electron/ion temperature, bulk velocity components, and turbulent velocity ${ }^{49,68}$ ); from these characteristics, a Thomson spectrum is computed for each ray using the approximations and code developed by Froula and coauthors. ${ }^{69}$ The overall simulated sum is then a weighted sum of the contributions from rays, where the weights include the effects of the probe beam shape and attenuation.

The experimental data for a shot corresponding to our 1Cl10ns simulation is shown in Figure 11(a). The Thomson scattering laser is on for $1 \mathrm{~ns}$ and probes a small $(\sim 50 \mu \mathrm{m}$ focal spot) volume between 32.5 and $33.5 \mathrm{~ns}$. The pair of ion-acoustic features is clearly visible, along with a stray-

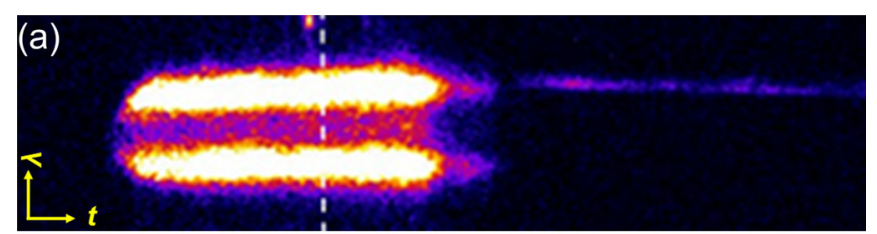

(b)

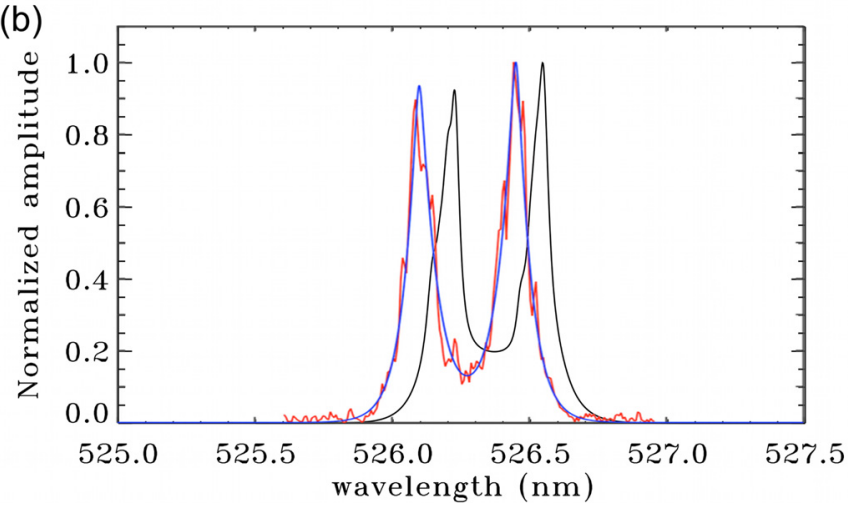

FIG. 11. (a) Time-streaked image of the Thomson scattered light, for an experiment corresponding to our $1 \mathrm{Cl10ns}$ case. The probe beam is on for a $1 \mathrm{~ns}$ interval between 32.5 and $33.5 \mathrm{~ns}$, targeted at the interaction region. The two ion features can be used to characterize the plasma properties. ${ }^{65}$ The white dashed line denotes the locus where the frequency lineout in panel (b) was taken. (b) Intensity profile at $t \sim 33 \mathrm{~ns}$. The red line is the experimental data, the blue line is an analytic fit without instrument noise, and the black line is the FLASH prediction. The shape of the ion features is in fairly good agreement with the experimental data, albeit there is a frequency offset between the features. This could be explained if the bulk velocity along the LoC found with FLASH were smaller by $\sim 50 \mathrm{~km} \mathrm{~s}^{-1}$ with respect to the one in the experimental data. light line at the laser wavelength $(526.5 \mathrm{~nm})$. The white dotted line denotes the locus where we extract a wavelength lineout to analyze the features (Figure 11(b)). The red line is the experimental data, the blue line is an analytic fit without instrument noise, and the black line is the FLASH prediction from the synthetic Thomson scattering diagnostic. The simulated spectrum agrees fairly well with the experimental result, in terms of shape, separation, and width of the ion features; these characteristics depend on the plasma properties discussed in Sec. V A. The discrepancy in terms of the position-which is defined by the Doppler shift due to the bulk velocity in the scattering volume-could be explained if the plasma in the experiment had a bulk velocity component along the LoC that is $\sim 50 \mathrm{~km} \mathrm{~s}^{-1}$ larger than the simulation. It is worth noting here that, due to the small volume probed by the Thomson scattering diagnostic, our correct prediction of the shape of the interaction region was crucial: had we focused the laser beam at the center of the target chamberwhere we would expect collision to occur-we would have missed the interaction region.

Proton radiography. To measure and characterize the magnetic field in the plasma, we use monoenergetic proton radiography. $^{70}$ This experimental diagnostic technique images magnetic fields using proton emission from the laserdriven implosion of a small $\mathrm{D}^{3} \mathrm{He}$ capsule. The capsule is located $1 \mathrm{~cm}$ away from the target chamber center (the center of our computational domain), and its implosion causes a quasi-isotropic emission of protons at $\sim 3$ and $\sim 14.7 \mathrm{MeV}$. The protons traverse the interaction region and interact only with the magnetic fields - as other physical effects such as collisions or kinetic effects are negligibly small. The protons are subsequently recorded on a detector (a CR39 plate) $28 \mathrm{~cm}$ away from the capsule. The deflection of a proton's path bears information on the morphology and strength of the magnetic fields that caused it; from the two-dimensional image, we can infer the path-integrated magnetic field, ${ }^{71,72}$ provided that the fields are not too strong.

We have implemented in FLASH a proton radiography synthetic diagnostic. The module fires protons towards the simulation domain and records their deflection due to electric and magnetic fields on a detector screen. By employing conical beams, the code can efficiently emulate a spherical sector of the isotropic emission, reducing considerably the computational cost of treating billions of protons - a typical proton yield of such a capsule implosion. Each proton is traced separately and is initialized with random velocity vectors in a spherical volume equal to the size of the capsule at bang time $(\sim 40 \mu \mathrm{m})$. The protons' deflections are calculated using the Lorentz force, assuming that the electric and magnetic fields do not change during the traversal of the domain by the protons. For each cell in the domain, the electric and magnetic fields are averaged from their staggered representations, ${ }^{41}$ and they are considered constant within each cell. The protons are collected on a screen, where we record their final position on the screen's coordinate system.

The simulated proton radiograph for case $1 \mathrm{Cl10 \textrm {ns }}$ at $31 \mathrm{~ns}$ is shown in Figure 12(a) for the $14.7 \mathrm{MeV}$ protons. The filamentary structure seen between the grids (grid A top left, grid $\mathrm{B}$ bottom right) is the result of proton deflection by the 

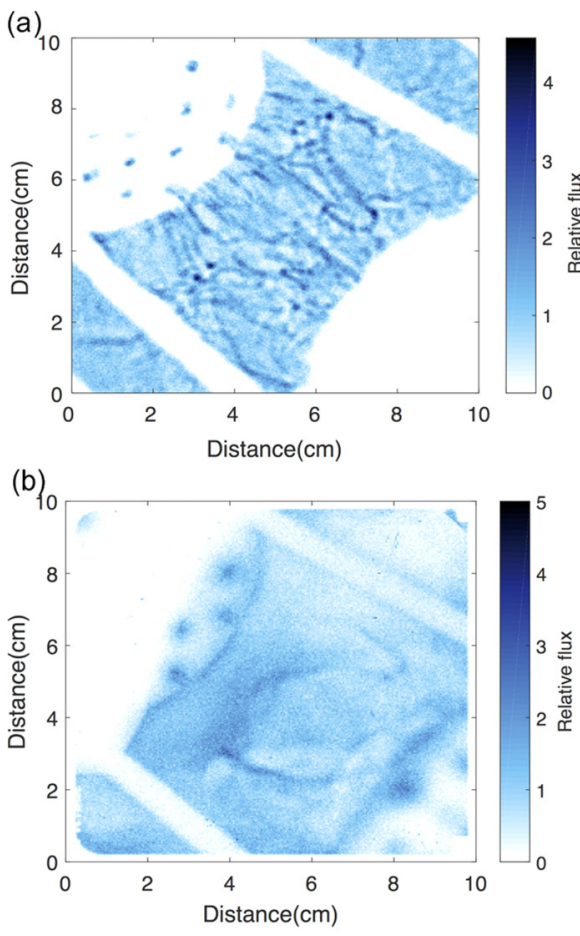

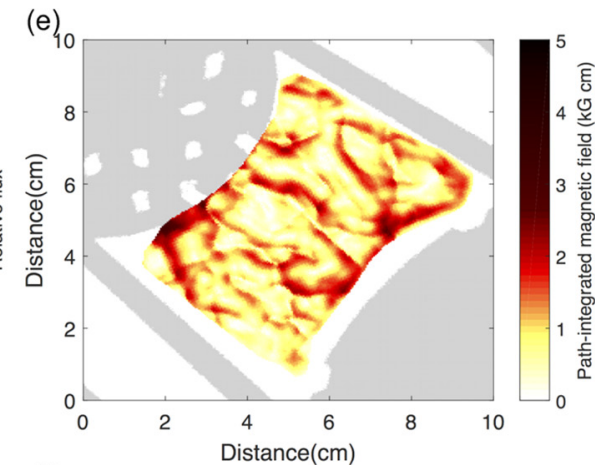

(d)
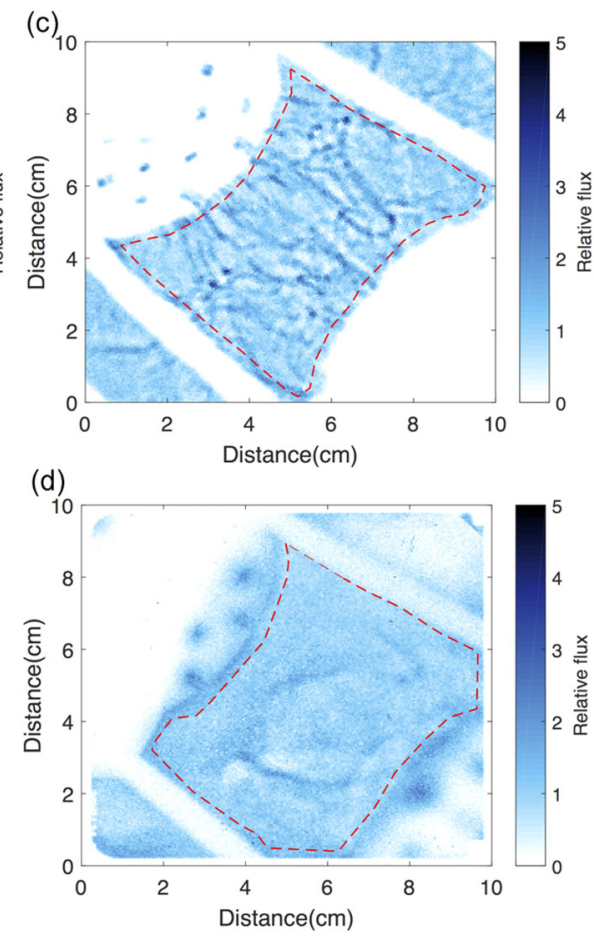

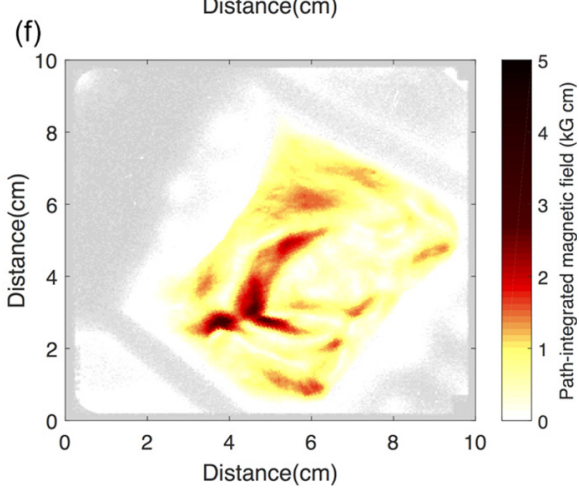

FIG. 12. Proton radiography images and path-integrated magnetic field reconstruction. (a) Simulated proton radiograph for case $1 \mathrm{Cl10}$ ns at $31 \mathrm{~ns}$. Shown is the relative proton flux for the $14.7 \mathrm{MeV}$ protons. The protons are obstructed by grids and rods, which are clearly visible. Grid A lies on the top left and grid B on the bottom right. The filamentary structures are the result of the magnetized turbulence that we have in the simulation. (b) Proton radiograph from an experimental configuration that corresponds to the case shown in panel (a). While a few filamentary structures exist, the experimental image exhibits more smearing, less small-scale structure, and systematic large-lengthscale variation in the proton flux. ${ }^{73}$ (c) Same as (a), but with a low-pass filter applied in the area denoted by the red dashed line, to remove the large-lengthscale variations. Since the synthetic image was made with an isotropic proton beam, the filtering has no effect. (d) Same as (b), but with a low-pass filter applied as in (c). The filamentary structures remain unaffected by the filtering. (e) Path-integrated magnetic field reconstruction on the synthetic proton radiograph shown in (c). (f) Same as (e), but for the experimental image in (d).

magnetic fields that develop in the turbulent interaction region, in the simulation. The synthetic image includes a number of smearing effects present in experimental images, such as the smearing due to the finite capsule size, the binning of protons, and Poisson noise. ${ }^{71}$ However, this list is not exhaustive and other plasma and instrument effects may affect the experimental proton radiograph. Experimental images suffer also from long lengthscale variations $\gtrsim 50 \%$ in the proton flux that should be taken into consideration in a quantitative analysis. ${ }^{73}$ The experimental proton radiograph that corresponds to the 1Cl10ns case is shown in Figure 12(b). This particular image shows only a few filamentary imprints on the CR39 plate. Moreover, it has more pronounced smearing and lacks the small-scale structure that we see in the simulated radiograph.

To evaluate quantitatively the field strength and topology from the experimental image and compare to the synthetic radiograph, we can apply either linear ${ }^{71}$ or non-linear ${ }^{72}$ reconstruction techniques to determine path-integrated fields. Since we are in the order-unity contrast regime, we utilize the latter. The first step in this analysis is to apply a low-pass filter on the proton radiographs to remove systematic large lengthscale variations, ${ }^{73}$ to which non-linear reconstruction techniques are sensitive (Figure 12(c) and 12(d)). This is performed on the areas denoted by the red dashed lines. The synthetic image remains unaltered due to the assumption of isotropic proton emission in the FLASH code, and the experimental image retains all its original sharp structures. Next, we apply the reconstruction to recover path-integrated magnetic field values in the image plane (Figure 12(e) and 12(f)). The number of path-integrated magnetic field structures is smaller in the experimental image, as expected following from the reduced number of filaments in the radiograph. We do however find agreement between the path-integrated magnetic field values $(\sim 3-5 \mathrm{kG} \mathrm{cm})$, which in turn can yield estimates of the magnetic field strength in the interaction region. ${ }^{30}$

\section{CONCLUSIONS}

The generation and amplification of magnetic fields observed in the universe is an ongoing research topic of modern astrophysics. While a number of mechanisms have been proposed to address generation of seed magnetic fields, ${ }^{4,7}$ the amplification is primarily attributed to turbulent dynamo, ${ }^{74-76}$ where the stochastic motions of the turbulent plasma can stretch and fold seed magnetic fields, amplifying them until they become dynamically significant. ${ }^{62}$ While a significant body of theoretical work exists on this process, turbulent dynamo has eluded a systematic study in the laboratory due to the large magnetic Reynolds numbers it requires to operate, especially in the small Pm regime. ${ }^{15}$

In this article, we described the numerical effort to design an experiment that could enable us to reach the turbulent dynamo regime. The results presented here highlight the advantages of using numerical modeling for experimental design and analysis. When combined with synthetic 
diagnostics, simulations can predict expected signals and be a crucial guide in determining the placement and timing of experimental diagnostics. Validated simulations produce data that can be analyzed quantitatively, allowing strong conclusions to be drawn from them.

The design of the experimental platform was based on our previous work (Figure 1) on laser-driven plasmas ${ }^{48,49,51}$ and the simulations described here. Our simulation campaign employed all the recently-developed HEDLP capabilities of the FLASH code and ANL's Mira BG/Q supercomputer. The configuration (Figure 2) that we designed consists of two diametrically opposed targets that are backlit with temporally stacked beams, which deliver $5 \mathrm{~kJ}$ of energy on each side; the beams drive a pair of colliding plasma flows that carry seed magnetic fields generated via a Biermann battery and propagate through a pair of grids, which destabilize them, introducing a driving scale; the flows meet at the center of the chamber to form a hot, turbulent interaction region (Figure 3), where we measure the plasma properties (Table II).

In the simulations, the turbulent plasma achieves sufficiently large $\mathrm{Rm}$ values that dynamo can act on the small seed fields and amplify them by a factor of $\sim 25$, reaching saturation within 1-2 eddy turnover times at the outer scale (Figures 5-7). The peak field values are of the order of $\sim 300-350 \mathrm{kG}$, with a magnetic-to-kinetic energy ratio of $\sim 1 \%-10 \%$, depending on the scale considered. In the modest dynamic range that we have in the simulations, the kinetic energy shows a Kolmogorov-like $k^{-5 / 3}$ power spectrum and the magnetic energy shows a $k^{-1}$ power spectrum, which are consistent with dynamo. This result provides upper bounds for the critical $\mathrm{Rm}$ value required by dynamo to operate: for the Re values achieved in the simulations, $\mathrm{Rm}_{c} \$ 900-1300$, consistent with the constraints derived for the small Pm regime. $^{15}$

The FLASH simulations were validated against a small subset of experimental data from our Omega experiments; ${ }^{30}$ we found good agreement in the propagation speed of the colliding fronts (Figure 8) and the collision timing (Figure 9). Moreover, the development of simulated diagnostics allowed us to compare synthetic vs. experimental data from the interaction region and find good agreement in terms of the shape of the interaction region (Figure 10) and the plasma properties (Figure 11). Nevertheless, some comparisons remain inconclusive: while FLASH predictions of a subsonic Kolmogorov MHD turbulence are consistent with the density power spectrum recovered from the X-rays, the filamentary structures seen in proton radiography are dissimilar, despite the apparent agreement in path-integrated magnetic field strength (Figure 12). To go beyond these validation-geared comparisons, a full analysis of the experimental dataset is needed; even if the simulation results indicate that the experimental platform is capable of demonstrating turbulent dynamo, the final outcome can only be decided by our experimental results, discussed in Ref. 30.

\section{ACKNOWLEDGMENTS}

We thank the anonymous reviewer whose constructive input improved the article's presentation. This work was supported in part at the University of Chicago by the U.S. Department of Energy (DOE) under Contract No. B523820 to the NNSA ASC/Alliances Center for Astrophysical Thermonuclear Flashes; the U.S. DOE NNSA ASC through the Argonne Institute for Computing in Science under field work proposal 57789; the U.S. DOE NNSA through the NLUF Grant No. DE-NA0002724; the U.S. DOE Office of Science through Grant No. DE-SC0016566; and the U.S. National Science Foundation under Grant Nos. PHY0903997 and PHY-1619573. Support from AWE plc., the Engineering and Physical Sciences Research Council (Grant Nos. EP/M022331/1; EP/N014472/1; and EP/N002644/1) and the Science and Technology Facilities Council of the United Kingdom is acknowledged. This work was supported in part by the National Research Foundation of Korea through grant 2016R1A5A1013277. Awards of computer time were provided by the DOE Innovative and Novel Computational Impact on Theory and Experiment (INCITE) and ASCR Leadership Computing Challenge (ALCC) programs. This research used resources of the Argonne Leadership Computing Facility at the Argonne National Laboratory, which is supported by the Office of Science of the U.S. Department of Energy under Contract No. DEAC02-06CH11357. The research and materials incorporated in this work were partially developed at the National Laser Users Facility at the University of Rochesters Laboratory for Laser Energetics, with financial support from the U.S. Department of Energy under Cooperative Agreement No. DE-NA0001944.

${ }^{1}$ E. N. Parker, Cosmical Magnetic Fields: Their Origin and Their Activity (Clarendon Press, Oxford, 1979).

${ }^{2}$ I. B. Zeldovich, A. A. Ruzmaikin, and D. D. Sokolov, "Magnetic fields in astrophysics," The Fluid Mechanics of Astrophysics and Geophysics (Gordon and Breach Science Publishers, New York, 1983), Vol. 3.

${ }^{3}$ H. K. Moffatt, Magnetic Field Generation in Electrically Conducting Fluids (Cambridge University Press, Cambridge, England, 1978).

${ }^{4}$ R. M. Kulsrud, R. Cen, J. P. Ostriker, and D. Ryu, Astrophys. J. 480, 481 (1997).

${ }^{5}$ L. Biermann, Z. Naturforsch. A 5, 65 (1950).

${ }^{6}$ E. S. Weibel, Phys. Rev. Lett. 2, 83 (1959).

${ }^{7}$ R. Schlickeiser and P. K. Shukla, Astrophys. J. Lett. 599, L57 (2003).

${ }^{8} \mathrm{~J}$. Larmor, in Proceedings of the 87th Meeting of Report of the British Association for the Advancement of Science (1919), p. 159.

${ }^{9}$ T. G. Cowling, Mon. Not. R. Astron. Soc. 94, 39 (1933).

${ }^{10} \mathrm{~S}$. I. Braginsky, "Self-excitation of a magnetic field during the motion of a highly conducting fluid," JETP 47, 1084 (1964) [Sov. Phys. JETP 20, 726 (1964)].

${ }^{11}$ W. M. Elsasser, Phys. Rev. 69, 106 (1946).

${ }^{12}$ G. Backus, Ann. Phys. 4, 372 (1958).

${ }^{13}$ F. Krause and K.-H. Raedler, Mean-Field Magnetohydrodynamics and Dynamo Theory (Pergamon Press, Oxford, 1980).

${ }^{14}$ S. M. Tobias, F. Cattaneo, and S. Boldyrev, in Ten Chapters in Turbulence, edited by P. A. Davidson, Y. Kaneda, and K. R. Sreenivasan (Cambridge University Press, Cambridge, 2013), Chap. 9, pp. 351-404.

${ }^{15}$ A. A. Schekochihin, A. Iskakov, S. Cowley, J. McWilliams, M. Proctor, and T. Yousef, New J. Phys. 9, 300 (2007).

${ }^{16} \mathrm{G}$. Verhille, N. Plihon, M. Bourgoin, P. Odier, and J.-F. Pinton, Space Sci. Rev. 152, 543 (2010).

${ }^{17}$ D. Biskamp, Magnetohydrodynamic Turbulence, edited by D. Biskamp (Cambridge University Press, Cambridge, UK, 2003), p. 310.

${ }^{18}$ A. Brandenburg and K. Subramanian, Phys. Rep. 417, 1 (2005).

${ }^{19}$ A. Lazarian, Int. J. Mod. Phys. D 15, 1099 (2006).

${ }^{20}$ R. M. Kulsrud and E. G. Zweibel, Rep. Prog. Phys. 71, 046901 (2008).

${ }^{21}$ A. Brandenburg and Å. Nordlund, Rep. Prog. Phys. 74, 046901 (2011). 
${ }^{22}$ A. Gailitis, O. Lielausis, S. Dement'ev, E. Platacis, A. Cifersons, G. Gerbeth, T. Gundrum, F. Stefani, M. Christen, H. Hänel, and G. Will, Phys. Rev. Lett. 84, 4365 (2000).

${ }^{23}$ A. Gailitis, O. Lielausis, E. Platacis, S. Dement'ev, A. Cifersons, G. Gerbeth, T. Gundrum, F. Stefani, M. Christen, and G. Will, Phys. Rev. Lett. 86, 3024 (2001).

${ }^{24}$ R. Monchaux, M. Berhanu, M. Bourgoin, M. Moulin, P. Odier, J.-F. Pinton, R. Volk, S. Fauve, N. Mordant, F. Pétrélis, A. Chiffaudel, F. Daviaud, B. Dubrulle, C. Gasquet, L. Marié, and F. Ravelet, Phys. Rev. Lett. 98, 044502 (2007).

${ }^{25}$ P. M. Bellan, Spheromaks: A Practical Application of Magnetohydrodynamic Dynamos and Plasma Self-Organization (Imperial College Press, London; Distributed by World Scientific Pub. Co., River Edge, NJ, 2000).

${ }^{26}$ D. D. Ryutov, R. Drake, J. Kane, E. Liang, B. Remington, and W. WoodVasey, Astrophys. J. 518, 821 (1999).

${ }^{27}$ D. D. Ryutov, R. P. Drake, and B. A. Remington, Astrophys. J. Suppl. Ser. 127, 465 (2000).

${ }^{28}$ B. A. Remington, D. Arnett, R. Paul, and H. Takabe, Science 284, 1488 (1999).

${ }^{29}$ G. Gregori, B. Reville, and F. Miniati, Phys. Rep. 601, 1 (2015).

${ }^{30}$ P. Tzeferacos, A. Rigby, A. Bott, A. R. Bell, R. Bingham, A. Casner, F. Cattaneo, E. M. Churazov, J. Emig, F. Fiuza, C. B. Forest, J. Foster, C. Graziani, J. Katz, M. Koenig, C.-K. Li, J. Meinecke, R. Petrasso, H.-S. Park, B. A. Remington, J. S. Ross, D. Ryu, D. Ryutov, T. G. White, B. Reville, F. Miniati, A. A. Schekochihin, D. Q. Lamb, D. H. Froula, and G. Gregori, "Laboratory evidence of dynamo amplification of magnetic fields in a turbulent plasma," Plasma Physics (submitted); e-print arXiv: 1702.03016

${ }^{31}$ B. A. Fryxell, K. Olson, P. Ricker, F. X. Timmes, M. Zingale, D. Q. Lamb, P. MacNeice, R. Rosner, J. W. Truran, and H. Tufo, Astrophys. J., Suppl. 131, 273 (2000).

${ }^{32}$ A. Dubey, K. Antypas, M. K. Ganapathy, L. B. Reid, K. Riley, D. Sheeler, A. Siegel, and K. Weide, Parallel Comp. 35, 512 (2009).

${ }^{33}$ The FLASH code is available at http://flash.uchicago.edu.

${ }^{34}$ P. Tzeferacos, M. Fatenejad, N. Flocke, C. Graziani, G. Gregori, D. Q. Lamb, D. Lee, J. Meinecke, A. Scopatz, and K. Weide, High Energy Density Phys. 17(Part A), 24 (2015).

${ }^{35}$ S. Braginskii, Rev. Plasma Phys. 1, 205 (1965).

${ }^{36}$ M. Haines, Plasma Phys. Controlled Fusion 28, 1705 (1986).

${ }^{37}$ M. Fatenejad, A. Bell, A. Benuzzi-Mounaix, R. Crowston, R. Drake, N. Flocke, G. Gregori, M. Koenig, C. Krauland, D. Lamb et al., High Energy Density Phys. 9, 172 (2013).

${ }^{38}$ C. Graziani, P. Tzeferacos, D. Lee, D. Q. Lamb, K. Weide, M. Fatenejad, and J. Miller, Astrophys. J. 802, 43 (2015).

${ }^{39}$ L. Spitzer, Physics of Fully Ionized Gases (Interscience, New York, 1962).

${ }^{40}$ D. Lee and A. E. Deane, J. Comput. Phys. 228, 952 (2009).

${ }^{41}$ D. Lee, J. Comput. Phys. 243, 269 (2013).

${ }^{42}$ P. Tzeferacos, M. Fatenejad, N. Flocke, G. Gregori, D. Lamb, D. Lee, J. Meinecke, A. Scopatz, and K. Weide, High Energy Density Phys. 8, 322 (2012).

${ }^{43}$ M. Gittings, R. Weaver, M. Clover, T. Betlach, N. Byrne, R. Coker, E. Dendy, R. Hueckstaedt, K. New, W. R. Oakes, D. Ranta, and R. Stefan, Computat. Sci. Discovery 1, 015005 (2008).

${ }^{44}$ The HYPRE library is available at https://computation-rnd.llnl.gov/linear_ solvers/software.php.

${ }^{45}$ T. B. Kaiser, Phys. Rev. E 61, 895 (2000).

${ }^{46}$ K. Falk, E. Gamboa, G. Kagan, D. Montgomery, B. Srinivasan, P. Tzeferacos, and J. Benage, Phys. Rev. Lett. 112, 155003 (2014).

${ }^{47}$ R. Yurchak, A. Ravasio, A. Pelka, S. Pikuz, Jr., E. Falize, T. Vinci, M. Koenig, B. Loupias, A. Benuzzi-Mounaix, M. Fatenejad et al., Phys. Rev. Lett. 112, 155001 (2014).
${ }^{48}$ J. Meinecke, H. Doyle, F. Miniati, A. Bell, R. Bingham, R. Crowston, R. Drake, M. Fatenejad, M. Koenig, Y. Kuramitsu et al., Nat. Phys. 10, 520 (2014).

${ }^{49}$ J. Meinecke, P. Tzeferacos, A. Bell, R. Bingham, R. Clarke, E. Churazov, R. Crowston, H. Doyle, R. P. Drake, R. Heathcote, M. Koenig, Y. Kuramitsu, C. Kuranz, D. Lee, M. MacDonald, C. Murphy, M. Notley, H.S. Park, A. Pelka, A. Ravasio, B. Reville, Y. Sakawa, W. Wan, N. Woolsey, R. Yurchak, F. Miniati, A. Schekochihin, D. Lamb, and G. Gregori, Proc. Natl. Acad. Sci. 112, 8211 (2015).

${ }^{50}$ C. K. Li, P. Tzeferacos, D. Lamb, G. Gregori, P. A. Norreys, M. J. Rosenberg, R. K. Follett, D. H. Froula, M. Koenig, F. H. Seguin, J. A. Frenje, H. G. Rinderknecht, H. Sio, A. B. Zylstra, R. D. Petrasso, P. A. Amendt, H. S. Park, B. A. Remington, D. D. Ryutov, S. C. Wilks, R. Betti, A. Frank, S. X. Hu, T. C. Sangster, P. Hartigan, R. P. Drake, C. C. Kuranz, S. V. Lebedev, and N. C. Woolsey, Nat. Commun. 7, 13081 (2016).

${ }^{51}$ G. Gregori, A. Ravasio, C. Murphy, K. Schaar, A. Baird, A. Bell, A. Benuzzi-Mounaix, R. Bingham, C. Constantin, R. Drake et al., Nature 481, 480 (2012).

${ }^{52}$ S. van den Bergh and W. W. Dodd, Astrophys. J. 162, 485 (1970).

${ }^{53}$ G. Golitsyn, Sov. Phys. Dokl. 5, 536 (1960).

${ }^{54}$ A. A. Schekochihin, S. C. Cowley, S. F. Taylor, J. L. Maron, and J. C. McWilliams, Astrophys. J. 612, 276 (2004).

${ }^{55}$ M. G. Haines, Phys. Rev. Lett. 78, 254 (1997).

${ }^{56}$ D. Biskamp and W.-C. Müller, Phys. Plasmas 7, 4889 (2000).

${ }^{57}$ Y. B. Zel'dovich and A. Ruzmaikin, Sov. Phys. JETP 51, 493 (1980); available at http://www.jetp.ac.ru/cgi-bin/dn/e_051_03_0493.pdf.

${ }^{58} \mathrm{PROPACEOS}$ is available at http://www.prism-cs.com/Software/ PROPACEOS/PROPACEOS.htm.

${ }^{59}$ P. Colella, J. Comput. Phys. 87, 171 (1990).

${ }^{60}$ P. Colella and P. R. Woodward, J. Comput. Phys. 54, 174 (1984).

${ }^{61} \mathrm{~S}$. Li, J. Comput. Phys. 203, 344 (2005).

${ }^{62}$ A. A. Schekochihin, S. C. Cowley, G. W. Hammett, J. L. Maron, and J. C. McWilliams, New J. Phys. 4, 84 (2002).

${ }^{63}$ A. A. Ruzmaikin and A. M. Shukurov, Astrophys. Space Sci. 82, 397 (1982).

${ }^{64}$ C. Federrath, G. Chabrier, J. Schober, R. Banerjee, R. S. Klessen, and D. R. G. Schleicher, Phys. Rev. Lett. 107, 114504 (2011).

${ }^{65}$ D. E. Evans and J. Katzenstein, Rep. Prog. Phys. 32, 207 (1969).

${ }^{66}$ E. Churazov, A. Vikhlinin, I. Zhuravleva, A. Schekochihin, I. Parrish, R. Sunyaev, W. Forman, H. Böhringer, and S. Randall, Mon. Not. R. Astron. Soc. 421, 1123 (2012).

${ }^{67}$ J. MacFarlane, I. Golovkin, P. Woodruff, D. Welch, B. Oliver, T. Mehlhorn, and R. Campbell, in Proceedings of the Inertial Fusion and Sciences Applications (2003), Vol. 457.

${ }^{68}$ N. A. Inogamov and R. A. Sunyaev, Astron. Lett. 29, 791 (2003).

${ }^{69}$ D. Froula, S. H. Glenzer, N. C. J. Luhmann, and J. Sheffield, Plasma Scattering of Electromagnetic Radiation (Academic, New York, 2010).

${ }^{70}$ C. Li, F. Séguin, J. Frenje, J. Rygg, R. Petrasso, R. Town, P. Amendt, S. Hatchett, O. Landen, A. Mackinnon, P. Patel, V. Smalyuk, T. Sangster, and J. Knauer, Phys. Rev. Lett. 97, 135003 (2006).

${ }^{71}$ C. Graziani, P. Tzeferacos, D. Q. Lamb, and C. Li, e-print arXiv: 1603.08617 [physics.plasm-ph].

${ }^{72}$ A. F. A. Bott, C. Graziani, P. Tzeferacos, D. Q. Lamb, G. Gregori, and A. Schekochihin, "Proton imaging of small-scale stochastic magnetic fields," Plasma Physics (submitted).

${ }^{73}$ M. J.-E. Manuel, A. B. Zylstra, H. G. Rinderknecht, D. T. Casey, M. J. Rosenberg, N. Sinenian, C. K. Li, J. A. Frenje, F. H. Séguin, and R. D. Petrasso, Rev. Sci. Instrum. 83, 063506 (2012).

${ }^{74}$ D. Ryu, H. Kang, J. Cho, and S. Das, Science 320, 909 (2008).

${ }^{75}$ A. A. Schekochihin and S. C. Cowley, Phys. Plasmas (1994-present) 13, 056501 (2006).

${ }^{76}$ K. Subramanian, A. Shukurov, and N. E. L. Haugen, Mon. Not. R. Astron. Soc. 366, 1437 (2006). 